\title{
ERROR ANALYSIS OF FINITE ELEMENT METHODS FOR SPACE-FRACTIONAL PARABOLIC EQUATIONS
}

\author{
BANGTI JIN, RAYTCHO LAZAROV, JOSEPH PASCIAK, AND ZHI ZHOU
}

\begin{abstract}
We consider an initial/boundary value problem for one-dimensional fractional-order parabolic equations with a space fractional derivative of RiemannLiouville type and order $\alpha \in(1,2)$. We study a spatial semidiscrete scheme with the standard Galerkin finite element method with piecewise linear finite elements, as well as fully discrete schemes based on the backward Euler method and CrankNicolson method. Error estimates in the $L^{2}(D)$ - and $H^{\alpha / 2}(D)$-norm are derived for the semidiscrete scheme, and in the $L^{2}(D)$-norm for the fully discrete schemes. These estimates are for both smooth and nonsmooth initial data, and are expressed directly in terms of the smoothness of the initial data. Extensive numerical results are presented to illustrate the theoretical results.
\end{abstract}

\section{INTRODUCTION}

We consider the following initial/boundary value problem for a space fractional-order parabolic differential equation (FPDE) for $u(x, t)$ :

$$
\begin{aligned}
u_{t}-{ }_{0}^{R} D_{x}^{\alpha} u & =f, \quad x \in D=(0,1), \quad 0<t \leq T, \\
u(0, t) & =u(1, t)=0, \quad 0<t \leq T, \\
u(x, 0) & =v, \quad x \in D,
\end{aligned}
$$

where $\alpha \in(1,2)$ is the order of the derivative, $f \in L^{2}\left(0, T ; L^{2}(D)\right)$, and ${ }_{0}^{R} D_{x}^{\alpha} u$ refers to the Riemann-Liouville fractional derivative of order $\alpha$, defined in (2.1) below, and $T>0$ is fixed. In case of $\alpha=2$, the fractional derivative ${ }_{0}^{R} D_{x}^{\alpha} u$ coincides with the usual secondorder derivative $u^{\prime \prime}[12$, and then model (1.1) recovers the classical diffusion equation.

The classical diffusion equation is often used to describe diffusion processes. The use of a Laplace operator in the equation rests on a Brownian motion assumption on the random motion of individual particles. However, over last few decades, a number of studies [1, 9, 14 have shown that anomalous diffusion, in which the mean square variances grows faster (superdiffusion) or slower (subdiffusion) than that in a Gaussian process, offers a superior fit to experimental data observed in some processes, e.g., viscoelastic materials, soil contamination, and underground water flow. In particular, at a microscopic level, the particle motion might be dependent, and can frequently take very large steps, following some heavy-tailed probability distribution. The long range correlation and large jumps can cause the underlying stochastic process to deviate significantly from Brownian motion for the classical diffusion process. Instead, a Levy process is considered to be more appropriate. The macroscopic counterpart is space fractional diffusion equations (SpFDEs) (1.1), and we refer to [1] for the derivation and relevant physical explanations. Numerous experimental studies have shown that SpFDEs can provide accurate description of the superdiffusion process.

Because of the extraordinary modeling capability of SpFDEs, their accurate numerical solution has become an important task. A number of numerical methods, prominently the finite difference method, have been developed for the time-dependent superdiffusion process in the literature. The finite difference scheme is usually based on a shifted Grünwald

Date: started May 21, 2013; today is July 25, 2018. 
formula for the Riemann-Liouville fractional derivative in space. In 16, 17, the stability, consistency and convergence were shown for the finite difference scheme with the CrankNicolson scheme in time. In these works, the convergence rates are provided under the a priori assumption that the solution $u$ to (1.1) is sufficiently smooth, which unfortunately is not justified in general, cf. Theorem 3.2

In this work, we develop a finite element method for (1.1). It is based on the variational formulation of the space fractional boundary value problem, initiated in 2, 3] and recently revisited in 11. We establish $L^{2}(D)$ - and $\widetilde{H}^{\alpha / 2}(D)$-norm error estimates for the space semidiscrete scheme, and $L^{2}(D)$-norm estimates for fully discrete schemes, using analytic semigroup theory 10]. Specifically, we obtained the following results. First, in Theorem 3.1 we establish the existence and uniqueness of a weak solution $u \in L^{2}\left(0, T ; \widetilde{H}^{\alpha / 2}(D)\right)$ of (1.1) (see Section 2 for the definitions of the space $\widetilde{H}^{\beta}(D)$ and the operator $A$ ) and in Theorem 3.2 show an enhanced regularity $u \in C\left((0, T] ; \widetilde{H}_{L}^{\alpha-1+\beta}(D)\right)$ with $\beta \in[0,1 / 2)$, for $v \in L^{2}(D)$. Second, in Theorems 4.2 and 4.1 we show that the semidiscrete finite element solution $u_{h}(t)$ with suitable discrete initial value $u_{h}(0)$ satisfies the a priori error bound

$\left\|u_{h}(t)-u(t)\right\|_{L^{2}(D)}+h^{\frac{\alpha}{2}-1+\beta}\left\|u_{h}(t)-u(t)\right\|_{\widetilde{H}^{\frac{\alpha}{2}(D)}} \leq C h^{\alpha-2+2 \beta} t^{l-1}\left\|A^{l} v\right\|_{L^{2}(D)}, l=0,1$,

with $h$ being the mesh size and any $\beta \in[0,1 / 2)$. Further we derived error estimates for the fully discrete solution $U^{n}$, with $\tau$ being the time step size and $t_{n}=n \tau$, for the backward Euler method and Crank-Nicolson method. For the backward Euler method, in Theorems 5.1 and 5.2 we establish the following error estimates

$$
\left\|u\left(t_{n}\right)-U^{n}\right\|_{L^{2}(D)} \leq C\left(h^{\alpha-2+2 \beta}+\tau\right) t_{n}^{l-1}\left\|A^{l} v\right\|_{L^{2}(D)} \quad l=0,1,
$$

and for the Crank-Nicolson method, in Theorems 5.3 and 5.4, we prove

$$
\left\|u\left(t_{n}\right)-U^{n}\right\|_{L^{2}(D)} \leq C\left(h^{\alpha-2+2 \beta}+\tau^{2} t_{n}^{-1}\right) t_{n}^{l-1}\left\|A^{l} v\right\|_{L^{2}(D)} .
$$

These error estimates cover both smooth and nonsmooth initial data and the bounds are directly expressed in terms of the initial data $v$. The case of nonsmooth initial data is especially interesting in inverse problems and optimal control.

The rest of the paper is organized as follows. In Section 2 we introduce preliminaries on fractional derivatives and related continuous and discrete variational formulations. Then in Section 3 we discuss the existence and uniqueness of a weak solution to (1.1) using a Galerkin procedure, and show the regularity pickup by the semigroup theory. Further, the properties of the discrete semigroup $E_{h}(t)$ are discussed. The error analysis for the semidiscrete scheme is carried out in Section 4 and that for fully discrete schemes based on the backward Euler method and the Crank-Nicolson method is provided in Section 5. Numerical results for smooth and nonsmooth initial data are presented in Section 6. Throughout, we use the notation $c$ and $C$, with or without a subscript, to denote a generic constant, which may change at different occurrences, but it is always independent of the solution $u$, time $t$, mesh size $h$ and time step size $\tau$.

\section{Fractional DERIVATIVES AND VARiational FORMUlation}

In this part, we describe fundamentals of fractional calculus, the variational problem for the source problem with a Riemann-Liouville fractional derivative, and discuss the finite element discretization.

2.1. Fractional derivatives. We first briefly recall the Riemann-Liouville fractional derivative. For any positive non-integer real number $\beta$ with $n-1<\beta<n, n \in \mathbb{N}$, the leftsided Riemann-Liouville fractional derivative ${ }_{0}^{R} D_{x}^{\beta} u$ of order $\beta$ of the function $u \in C^{n}[0,1]$ is defined by [12, pp. 70]:

$$
{ }_{0}^{R} D_{x}^{\beta} u=\frac{d^{n}}{d x^{n}}\left({ }_{0} I_{x}^{n-\beta} u\right) .
$$


Here ${ }_{0} I_{x}^{\gamma}$ for $\gamma>0$ is the left-sided Riemann-Liouville fractional integral operator of order $\gamma$ defined by

$$
\left({ }_{0} I_{x}^{\gamma} f\right)(x)=\frac{1}{\Gamma(\gamma)} \int_{0}^{x}(x-t)^{\gamma-1} f(t) d t
$$

where $\Gamma(\cdot)$ is Euler's Gamma function defined by $\Gamma(x)=\int_{0}^{\infty} t^{x-1} e^{-t} d t$. The right-sided versions of fractional-order integral and derivative are defined analogously, i.e.,

$$
\left({ }_{x} I_{1}^{\gamma} f\right)(x)=\frac{1}{\Gamma(\gamma)} \int_{x}^{1}(x-t)^{\gamma-1} f(t) d t \quad \text { and } \quad{ }_{x}^{R} D_{1}^{\beta} u=(-1)^{n} \frac{d^{n}}{d x^{n}}\left({ }_{x} I_{1}^{n-\beta} u\right) .
$$

Now we introduce some function spaces. For any $\beta \geq 0$, we denote $H^{\beta}(D)$ to be the Sobolev space of order $\beta$ on the unit interval $D=(0,1)$, and $\widetilde{H}^{\beta}(D)$ to be the set of functions in $H^{\beta}(D)$ whose extension by zero to $\mathbb{R}$ are in $H^{\beta}(\mathbb{R})$. Analogously, we define $\widetilde{H}_{L}^{\beta}(D)$ (respectively, $\widetilde{H}_{R}^{\beta}(D)$ ) to be the set of functions $u$ whose extension by zero $\tilde{u}$ is in $H^{\beta}(-\infty, 1)$ (respectively, $H^{\beta}(0, \infty)$ ). Here for $u \in \widetilde{H}_{L}^{\beta}(D)$, we set $\|u\|_{\widetilde{H}_{L}^{\beta}(D)}:=\|\tilde{u}\|_{H^{\beta}(-\infty, 1)}$ with an analogous definition for the norm in $\widetilde{H}_{R}^{\beta}(D)$. The fractional derivative operator ${ }_{0}^{R} D_{x}^{\beta}$ is well defined for functions in $C^{n}[0,1]$, and can be extended continuously from $\widetilde{H}_{L}^{\alpha}(D)$ to $L^{2}(D)$ ([2, Lemma 2.6], 11, Theorem 2.2]).

2.2. Variational formulation and its discretization. Now we recall the variational formulation for the source problem

$$
-{ }_{0}^{R} D_{x}^{\alpha} u=f,
$$

with $u(0)=u(1)=0$, and $f \in L^{2}(D)$. The proper variational formulation is given by 11]: find $u \in U \equiv \widetilde{H}^{\alpha / 2}(D)$ such that

$$
A(u, \psi)=\langle f, \psi\rangle \quad \forall \psi \in U
$$

where the sesquilinear form $A(\cdot, \cdot)$ is given by

$$
A(\varphi, \psi)=-\left({ }_{0}^{R} D_{x}^{\alpha / 2} \varphi,{ }_{x}^{R} D_{1}^{\alpha / 2} \psi\right) .
$$

It is known ([2, Lemma 3.1], 11, Lemma 4.2]) that the sesquilinear form $A(\cdot, \cdot)$ is coercive on the space $U$, i.e., there is a constant $c_{0}$ such that for all $\psi \in U$

$$
\Re A(\psi, \psi) \geq c_{0}\|\psi\|_{U}^{2},
$$

where $\Re$ denotes taking the real part, and continuous on $U$, i.e., for all $\varphi, \psi \in U$

$$
|A(\varphi, \psi)| \leq C_{0}\|\varphi\|_{U}\|\psi\|_{U} .
$$

Then by Riesz representation theorem, there exists a unique bounded linear operator $\widetilde{A}: \widetilde{H}^{\alpha / 2}(D) \rightarrow H^{-\alpha / 2}(D)$ such that

$$
A(\varphi, \psi)=\langle\widetilde{A} \varphi, \psi\rangle, \quad \forall \varphi, \psi \in \widetilde{H}^{\alpha / 2}(D) .
$$

Define $D(A)=\left\{\psi \in \widetilde{H}^{\alpha / 2}(D): \widetilde{A} \psi \in L^{2}(D)\right\}$ and an operator $A: D(A) \rightarrow L^{2}(D)$ by

$$
A(\varphi, \psi)=(A \varphi, \psi), \varphi \in D(A), \psi \in \widetilde{H}^{\alpha / 2}(D) .
$$

Remark 2.1. The domain $D(A)$ has a complicated structure: it consists of functions of the form $I_{0}^{\alpha} f-\left(I_{0}^{\alpha} f\right)(1) x^{\alpha-1}$, where $f \in L^{2}(D)$ 11. The term $x^{\alpha-1} \in \widetilde{H}_{L}^{\alpha-1+\beta}(D)$, $\beta \in[0,1 / 2)$, appears because it is in the kernel of the operator ${ }_{0}^{R} D_{x}^{\alpha}$. Hence, $D(A) \subset$ $\widetilde{H}_{L}^{\alpha-1+\beta}(D) \cap \widetilde{H}^{\alpha / 2}(D)$ and it is dense in $L^{2}(D)$.

The next result shows that the linear operator $A$ is sectorial, which means that

(1) the resolvent set $\rho(A)$ contains the sector $\Sigma_{\theta}=\{z: \theta \leq|\arg z| \leq \pi\}$ for $\theta \in$ $(0, \pi / 2)$

(2) $\left\|(\lambda I-A)^{-1}\right\| \leq M /|\lambda|$ for $\lambda \in \Sigma_{\theta}$ and some constant $M$. 
Then we have the following important lemma (cf. [10, pp. 94, Theorem 3.6]), for which we sketch a proof for completeness.

Lemma 2.1. The linear operator $A$ defined in (2.5) is sectorial on $L^{2}(D)$.

Proof. For all $\varphi \in D(A)$, we obtain by (2.3) and (2.4)

$$
|(A \varphi, \varphi)| \leq C_{0}\|\varphi\|_{\widetilde{H}^{\alpha / 2}(D)}^{2} \leq \frac{C_{0}}{c_{0}} \Re(A \varphi, \varphi) .
$$

Thus $\mathcal{N}(A)$, the numerical range of $A$, which is defined by

$$
\mathcal{N}(A)=\left\{(A \varphi, \varphi): \varphi \in D(A) \text { and }\|\varphi\|_{L^{2}(D)}=1\right\},
$$

is included in the sector $\Sigma_{0}=\left\{z: 0 \leq|\arg (z)| \leq \delta_{0}\right\}$, with $\delta_{0}=\arccos \left(c_{0} / C_{0}\right)$.

Now we choose $\delta_{1} \in\left(\delta_{0}, \frac{\pi}{2}\right)$ and set $\Sigma_{\delta_{1}}=\left\{z: \delta_{1} \leq|\arg (z)| \leq \pi\right\}$. Then by 8. p. 310, Propositon C.3.1], the resolvent set $\rho(A)$ contains $\Sigma_{\delta_{1}}$ and for all $\lambda \in \Sigma_{\delta_{1}}$

$$
\left\|(\lambda I-A)^{-1}\right\| \leq \frac{1}{\operatorname{dist}(\lambda, \overline{\mathcal{N}(A)})} \leq \frac{1}{\operatorname{dist}\left(\lambda, \Sigma_{0}\right)} \leq \frac{1}{\sin \left(\delta_{1}-\delta_{0}\right)} \frac{1}{|\lambda|} .
$$

That completes the proof of this lemma.

The next corollary is an immediate consequence of Lemma 2.1 .

Corollary 2.1. The linear operator $A$ is the infinitesimal generator of an analytic semigroup $E(t)=e^{-A t}$ on $L^{2}(D)$.

Proof. It follows directly from Lemma 2.1 and standard semigroup theory, cf., 10, Theorem 3.4, Proposition 3.9 and Theorem 3.19].

2.3. Finite element discretization. We introduce a finite element approximation based on an equally spaced partition of the interval $D$. We let $h=1 / m$ be the mesh size with $m>1$ being a positive integer, and consider the nodes $x_{j}=j h, j=0, \ldots, m$. We then define $U_{h}$ to be the set of continuous functions in $U$ which are linear when restricted to the subintervals $\left[x_{i}, x_{i+1}\right], i=0, \ldots, m-1$, i.e.,

$$
U_{h}=\left\{\chi \in C_{0}(\bar{D}): \chi \text { is linear over }\left[x_{i}, x_{i+1}\right], i=0, \ldots, m\right\} .
$$

We define the discrete operator $A_{h}: U_{h} \rightarrow U_{h}$ by

$$
\left(A_{h} \varphi, \chi\right)=A(\varphi, \chi), \quad \forall \varphi, \chi \in U_{h} .
$$

The lemma below is a direct corollary of properties (2.3) and (2.4) of the bilinear form $A(\cdot, \cdot)$ :

Lemma 2.2. The discrete operator $A_{h}$ satisfies

$$
\begin{aligned}
\Re\left(A_{h} \psi, \psi\right) & \geq c_{0}\|\psi\|_{\widetilde{H}^{\alpha / 2}(D)}^{2}, \quad \psi \in U_{h}, \\
\left|\left(A_{h} \varphi, \psi\right)\right| & \leq C_{0}\|\varphi\|_{\widetilde{H}^{\alpha / 2}(D)}\|\psi\|_{\widetilde{H}^{\alpha / 2}(D)}, \quad \varphi, \psi \in U_{h} .
\end{aligned}
$$

Remark 2.2. By Lemma 2.2 and repeating the argument in the proof of Lemma 2.1, we can show that $A_{h}$ is a sectorial operator on $U_{h}$ with the same constant as $A$.

Next we recall the Ritz projection $R_{h}: \widetilde{H}^{\alpha / 2}(D) \rightarrow U_{h}$ and the $L^{2}(D)$-projection $P_{h}: L^{2}(D) \rightarrow U_{h}$, respectively, defined by

$$
\begin{aligned}
A\left(R_{h} \psi, \chi\right) & =A(\psi, \chi) \quad \forall \psi \in \widetilde{H}^{\alpha / 2}(D), \chi \in U_{h}, \\
\left(P_{h} \varphi, \chi\right) & =(\varphi, \chi) \quad \forall \varphi \in L^{2}(D), \chi \in U_{h} .
\end{aligned}
$$

We shall also need the adjoint problem in the error analysis. Similar to (2.5), we define the adjoint operator $A^{*}$ as

$$
A(\varphi, \psi)=\left(\varphi, A^{*} \psi\right), \quad \forall \varphi \in \widetilde{H}^{\alpha / 2}(D), \psi \in D\left(A^{*}\right),
$$


where the domain $D\left(A^{*}\right)$ of $A^{*}$ satisfies $D\left(A^{*}\right) \subset \widetilde{H}_{R}^{\alpha-1+\beta}(D) \cap \widetilde{H}^{\alpha / 2}(D)$ and it is dense in $L^{2}(D)$. Further, the discrete analogue $A_{h}^{*}$ of $A^{*}$ is defined by

$$
A(\varphi, \psi)=\left(\varphi, A_{h}^{*} \psi\right), \quad \forall \varphi, \psi \in U_{h} .
$$

\section{VARIATIONAL FORMULATION OF FRACTIONAL-ORDER PARABOLIC PROBLEM}

The variational formulation of problem (1.1) is to find $u(t) \in U$ such that

$$
\left(u_{t}, \varphi\right)+A(u, \varphi)=(f, \varphi) \quad \forall \varphi \in U,
$$

and $u(0)=v$. We shall establish the well-posedness of the variational formulation 3.1 using a Galerkin procedure, and an enhanced regularity estimate via analytic semigroup theory. Further, the properties of the discrete semigroup are discussed.

3.1. Existence and uniqueness of the weak solution. First we state an existence and uniqueness of a weak solution, following a Galerkin procedure 44. To this end, we choose an orthogonal basis $\left\{\omega_{k}(x)=\sqrt{2} \sin k \pi x\right\}$ in both $L^{2}(D)$ and $H_{0}^{1}(D)$ and orthonormal in $L^{2}(D)$. In particular, by the construction, the $L^{2}(D)$-orthogonal projection operator $P$ into $\operatorname{span}\left\{\omega_{k}\right\}$ is stable in both $L^{2}(D)$ and $H_{0}^{1}(D)$, and by interpolation, it is also stable in $\widetilde{H}^{\beta}(D)$ for any $\beta \in[0,1]$. Now we fix a positive integer $m$, and look for a solution $u_{m}(t)$ of the form

$$
u_{m}(t):=\sum_{k=1}^{m} c_{k}(t) \omega_{k}
$$

such that for $k=1,2 \ldots, m$

$$
c_{k}(0)=\left(v, \omega_{k}\right), \quad\left(u_{m}^{\prime}, \omega_{k}\right)+A\left(u_{m}, \omega_{k}\right)=\left(f, \omega_{k}\right), \quad 0 \leq t \leq T .
$$

The existence and uniqueness of $u_{m}$ follows directly from the standard theory for ordinary differential equation systems. With the finite-dimensional approximation $u_{m}$ at hand, one can deduce the following existence and uniqueness result. The proof is rather standard, and it is given in Appendix $\mathrm{A}$ for completeness.

Theorem 3.1. Let $f \in L^{2}\left(0, T ; L^{2}(D)\right)$ and $v \in L^{2}(D)$. Then there exists a unique weak solution $u \in L^{2}\left(0, T ; \widetilde{H}^{\alpha / 2}(D)\right)$ of (3.1).

Now we study the regularity of the solution $u$ using semigroup theory [10. By Corollary 2.1 and the classical semigroup theory, the solution $u$ to the initial boundary value problem (1.1) with $f \equiv 0$ can be represented as

$$
u(t)=E(t) v,
$$

where $E(t)=e^{-t A}$ is the semigroup generated by the sectorial operator $A$, cf. Corollary 2.1. Then we have an improved regularity by [15, p. 104, Corolary 1.5].

Theorem 3.2. For every $v \in L^{2}(D)$, the homogeneous initial-boundary value problem (3.1) (with $f=0)$ has a unique solution $u(x, t) \in C\left([0, T] ; L^{2}(D)\right) \cap C((0, T] ; D(A))$.

Further, we have the following $L^{2}(D)$ estimate.

Lemma 3.1. There is a constant $C$ such that

$$
\left\|A^{\gamma} E(t) \psi\right\|_{L^{2}(D)} \leq C t^{-\gamma}\|\psi\|_{L^{2}(D)} .
$$

Proof. The cases $\gamma=0$ and $\gamma=1$ have been proved in [18, pp. 91, Theorem 6.4 (iii)]. With the contour $\Gamma=\left\{z: z=\rho e^{ \pm \mathrm{i} \delta_{1}}, \rho \geq 0\right\}$, the case of $\gamma \in(0,1)$ follows by

$$
\begin{aligned}
\left\|A^{\gamma} E(t) \psi\right\|_{L^{2}(D)} & =\left\|\frac{1}{2 \pi \mathrm{i}} \int_{\Gamma} z^{\gamma} e^{-z t} R(z ; A) \psi d z\right\|_{L^{2}(D)} \\
& \leq C\|\psi\|_{L^{2}(D)} \int_{0}^{\infty} \rho^{\gamma-1} e^{-\rho t} d \rho \leq C t^{-\gamma}\|\psi\|_{L^{2}(D)} .
\end{aligned}
$$


3.2. Properties of the semigroup $E_{h}(t)$. Let $E_{h}(t)=e^{-A_{h} t}$ be the semigroup generated by the operator $A_{h}$. Then it satisfies a discrete analogue of Lemma 3.1

Lemma 3.2. There exists a constant $C>0$ such that for $\chi \in U_{h}$

$$
\left\|A_{h}^{\gamma} E_{h}(t) \chi\right\|_{L^{2}(D)} \leq C t^{-\gamma}\|\chi\|_{L^{2}(D)} \text {. }
$$

Proof. It follows directly from Remark 2.2 and Lemma 3.1

Last we recall the Dunford-Taylor spectral representation of a rational function $r\left(A_{h}\right)$ of the operator $A_{h}$, when $r(z)$ is bounded in a sector in the right half plane 18, Lemma $9.1]$.

Lemma 3.3. Let $r(z)$ be a rational function that is bounded for $|\arg z| \leq \delta_{1},|z| \geq \epsilon>0$, and for $|z| \geq R$. Then if $\epsilon>0$ is so small that $\{z:|z| \leq \epsilon\} \subset \rho\left(A_{h}\right)$, we have

$$
r\left(A_{h}\right)=r(\infty) I+\frac{1}{2 \pi \mathrm{i}} \int_{\Gamma_{\epsilon} \cup \Gamma_{\epsilon}^{R} \cup \Gamma^{R}} r(z) R\left(z ; A_{h}\right) d z,
$$

where $R\left(z ; A_{h}\right)=\left(z I-A_{h}\right)^{-1}$ is the resolvent operator, $\Gamma_{\epsilon}^{R}=\left\{z:|\arg z|=\delta_{1}, \epsilon \leq|z| \leq\right.$ $R\}, \Gamma_{\epsilon}=\left\{z:|z|=\epsilon,|\arg z| \leq \delta_{1}\right\}$, and $\Gamma^{R}=\left\{z:|z|=R, \delta_{1} \leq|\arg z| \leq \pi\right\}$, and with the closed path of integration oriented in the negative sense.

Remark 3.1. The representation in Lemma 3.3 holds true for any function $f(z)$ which is analytic in a neighborhood of $\left\{z:|\arg z| \leq \delta_{1},|z| \geq \epsilon\right\}$, including at $z=\infty$.

\section{Error estimates for semidiscrete Galerkin FEM}

In this section, we derive $L^{2}(D)$ - and $\widetilde{H}^{\alpha / 2}(D)$-norm error estimates for the semidiscrete Galerkin FEM: find $u_{h}(t) \in U_{h}$ such that

$$
\left(u_{h, t}, \varphi\right)+A\left(u_{h}, \varphi\right)=(f, \varphi), \quad \forall \varphi \in U_{h}, T \geq t>0 . \quad u_{h}(0)=v_{h},
$$

where $v_{h} \in U_{h}$ is an approximation to the initial data $v$. We shall discuss the case of smooth and nonsmooth initial data, i.e. $v \in D(A)$ and $v \in L^{2}(D)$, separately.

4.1. Error estimate for nonsmooth initial data. First we consider nonsmooth initial data, i.e., $v \in L^{2}(D)$. We follow the approach due to Fujita and Suzuki [6]. First, we have the following important lemma. Here we shall use the constant $\delta_{1}$ and the contour $\Gamma=\left\{z: z=\rho e^{ \pm \mathrm{i} \delta_{1}}, \rho \geq 0\right\}$ defined in the proof of Lemma 2.1.

Lemma 4.1. There exists a constant $C>0$ such that for any $\varphi \in \widetilde{H}^{\alpha / 2}(D)$ and $z \in \Gamma$

$$
|z|\|\varphi\|_{L^{2}(D)}^{2}+\|\varphi\|_{\widetilde{H}^{\alpha / 2}(D)}^{2} \leq C\left|z\|\varphi\|_{L^{2}(D)}^{2}-A(\varphi, \varphi)\right| .
$$

Proof. We use the notation $\delta_{0}$ and $\delta_{1}$ from the proof of Lemma 2.1. Then we choose $\delta^{\prime}$ such that $\delta^{\prime} \in\left(\delta_{0}, \delta_{1}\right)$ and let $c^{\prime}=C_{0} \cos \delta^{\prime}$, cf. Fig. 1). By setting $\gamma=c_{0}-c^{\prime}>0$, we have

$$
\Re A(\varphi, \varphi)-\gamma\|\varphi\|_{\widetilde{H}^{\alpha / 2}(D)}^{2} \geq c^{\prime}\|\varphi\|_{\widetilde{H}^{\alpha / 2}(D)}^{2} \geq \cos \delta^{\prime}|A(\varphi, \varphi)| .
$$

By dividing both sides by $\|\varphi\|_{L^{2}(D)}^{2}$, this yields

$$
|A(\varphi, \varphi)| /\|\varphi\|_{L^{2}(D)}^{2} \in \Sigma_{\varphi}=\left\{z:\left|\arg \left(z-\gamma\|\varphi\|_{\widetilde{H}^{\alpha / 2}(D)}^{2} /\|\varphi\|_{L^{2}(D)}^{2}\right)\right| \leq \delta^{\prime}\right\} .
$$

Note that for $z \in \Gamma$, there holds, cf. Fig. 1(a)

$$
\operatorname{dist}\left(z, \Sigma_{\varphi}\right) \geq|z| \sin \left(\delta_{1}-\delta^{\prime}\right)+\gamma\|\varphi\|_{\widetilde{H}^{\alpha / 2}(D)}^{2} /\|\varphi\|_{L^{2}(D)}^{2} \sin \delta^{\prime} .
$$

Consequently, for $z \in \Gamma$ we get

$$
\begin{aligned}
\left|z\|\varphi\|_{L^{2}(D)}^{2}-A(\varphi, \varphi)\right| & \geq\|\varphi\|_{L^{2}(D)}^{2} \operatorname{dist}\left(z, \Sigma_{\varphi}\right) \\
& \geq|z|\|\varphi\|_{L^{2}(D)}^{2} \sin \left(\delta_{1}-\delta^{\prime}\right)+\gamma\|\varphi\|_{\widetilde{H}^{\alpha / 2}(D)}^{2} \sin \delta^{\prime} \\
& \geq \frac{1}{C}\left(|z|\|\varphi\|_{L^{2}(D)}^{2}+\|\varphi\|_{\widetilde{H}^{\alpha / 2}(D)}^{2}\right),
\end{aligned}
$$


and this completes the proof.

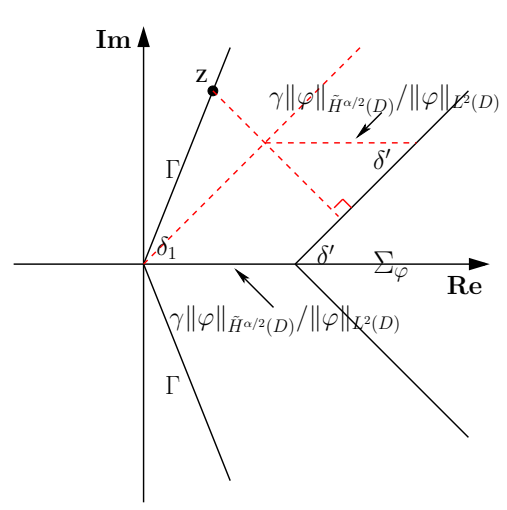

(a)

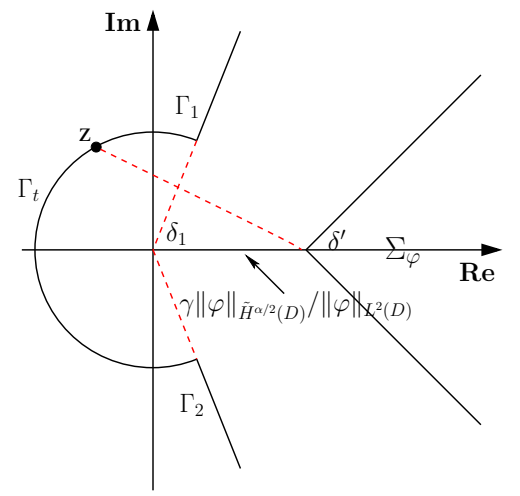

(b)

FIGURE 1. Integration path $\Sigma_{\delta_{1}}$ and $\Sigma_{\varphi}$ for (a) nonsmooth and (b) smooth initial data.

The next result gives estimates on the resolvent $R(z ; A) v$ and its discrete analogue.

Lemma 4.2. Let $v \in L^{2}(D), z \in \Gamma, w=R(z ; A) v$, and $w_{h}=R\left(z ; A_{h}\right) P_{h} v$. Then for $\beta \in[0,1 / 2)$, there holds

$$
\left\|w_{h}-w\right\|_{L^{2}(D)}+h^{\alpha / 2-1+\beta}\left\|w_{h}-w\right\|_{\widetilde{H}^{\alpha / 2}(D)} \leq C h^{\alpha-2+2 \beta}\|v\|_{L^{2}(D)} .
$$

Proof. By the definition, $w$ and $w_{h}$ should respectively satisfy

$$
\begin{aligned}
z(w, \varphi)-A(w, \varphi) & =(v, \varphi), \quad \forall \varphi \in U, \\
z\left(w_{h}, \varphi\right)-A\left(w_{h}, \varphi\right) & =(v, \varphi), \quad \forall \varphi \in U_{h} .
\end{aligned}
$$

Upon subtracting these two identities, it gives an orthogonality relation for $e=w-w_{h}$ :

$$
z(e, \varphi)-A(e, \varphi)=0, \quad \forall \varphi \in U_{h} .
$$

This and Lemma 4.1 imply that for any $\chi \in U_{h}$

$$
\begin{aligned}
|z|\|e\|_{L^{2}(D)}^{2}+\|e\|_{\widetilde{H}^{\alpha / 2}(D)}^{2} & \leq C\left|z\|e\|_{L^{2}(D)}^{2}-A(e, e)\right| \\
& =C|z(e, w-\chi)-A(e, w-\chi)| .
\end{aligned}
$$

By taking $\chi=\pi_{h} w$, the finite element interpolant of $w$, and the Cauchy-Schwarz inequality, we obtain

$$
\begin{aligned}
|z|\|e\|_{L^{2}(D)}^{2}+\|e\|_{\widetilde{H}^{\alpha / 2}(D)}^{2} \leq C & \left(|z| h^{\alpha / 2}\|e\|_{L^{2}(D)}\|w\|_{\widetilde{H}^{\alpha / 2}(D)}\right. \\
& \left.+h^{\alpha / 2-1+\beta}\|e\|_{\widetilde{H}^{\alpha / 2}(D)}\|w\|_{H^{\alpha-1+\beta}(D)}\right) .
\end{aligned}
$$

Appealing again to Lemma 4.1 with the choice $\varphi=w$, we arrive at

$$
|z|\|w\|_{L^{2}(D)}^{2}+\|w\|_{\widetilde{H}^{\alpha / 2}(D)}^{2} \leq C|((z I-A) w, w)| \leq C\|v\|_{L^{2}(D)}\|w\|_{L^{2}(D)} .
$$

Consequently

$$
\|w\|_{L^{2}(D)} \leq C|z|^{-1}\|v\|_{L^{2}(D)} \quad \text { and } \quad\|w\|_{\widetilde{H}^{\alpha / 2}(D)} \leq C|z|^{-1 / 2}\|v\|_{L^{2}(D)} .
$$

It remains to bound $\|w\|_{H^{\alpha-1+\beta}(D)}$. To this end, we deduce from (4.6) that

$$
\begin{aligned}
\|w\|_{H^{\alpha-1+\beta}(D)} & \leq C\|A w\|_{L^{2}(D)}=C\|(A-z I+z I) R(z ; A) v\|_{L^{2}(D)} \\
& \leq C\left(\|v\|_{L^{2}(D)}+|z|\|w\|_{L^{2}(D)}\right) \leq C\|v\|_{L^{2}(D)} .
\end{aligned}
$$


It follows from this and 4.5 that

$$
|z|\|e\|_{L^{2}(D)}^{2}+\|e\|_{\widetilde{H}^{\alpha / 2}(D)}^{2} \leq C h^{\alpha / 2-1+\beta}\|v\|\left(|z|^{1 / 2}\|e\|_{L^{2}(D)}+\|e\|_{\widetilde{H}^{\alpha / 2}(D)}\right),
$$

i.e.,

$$
|z|\|e\|_{L^{2}(D)}^{2}+\|e\|_{\widetilde{H}^{\alpha / 2}(D)}^{2} \leq C h^{\alpha-2+2 \beta}\|v\|_{L^{2}(D)}^{2} .
$$

from which follows directly the $\widetilde{H}^{\alpha / 2}(D)$-norm of the error $e$. Next we deduce the $L^{2}(D)$ norm of the error $e$ by a duality argument: given $\varphi \in L^{2}(D)$, we define $\psi$ and $\psi_{h}$ respectively by

Then by duality

$$
\psi=R\left(z ; A^{*}\right) \varphi \quad \text { and } \quad \psi_{h}=R\left(z ; A_{h}^{*}\right) P_{h} \varphi
$$

$$
\|e\|_{L^{2}(D)} \leq \sup _{\varphi \in L^{2}(D)} \frac{|(e, \varphi)|}{\|\varphi\|_{L^{2}(D)}}=\sup _{\varphi \in L^{2}(D)} \frac{|z(e, \psi)-A(e, \psi)|}{\|\varphi\|_{L^{2}(D)}} .
$$

Meanwhile it follows from (4.4) and (4.7) that

$$
\begin{aligned}
|z(e, \psi)-A(e, \psi)| & =\left|z\left(e, \psi-\psi_{h}\right)-A\left(e, \psi-\psi_{h}\right)\right| \\
& \leq|z|\|e\|_{L^{2}(D)}\left\|\psi-\psi_{h}\right\|_{L^{2}(D)}+C\|e\|_{\widetilde{H}^{\alpha / 2}(D)}\left\|\psi-\psi_{h}\right\|_{\widetilde{H}^{\alpha / 2}(D)} \\
& \leq C h^{\alpha-2+2 \beta}\|v\|_{L^{2}(D)}\|\varphi\|_{L^{2}(D)} .
\end{aligned}
$$

This completes proof of the lemma.

Now we can state our first error estimate.

Theorem 4.1. Let $u$ and $u_{h}$ be solutions of problem (3.1) and (4.1) with $v \in L^{2}(D)$ and $v_{h}=P_{h} v$, respectively. Then for $t>0$, there holds for any $\beta \in[0,1 / 2)$ :

$$
\left\|u(t)-u_{h}(t)\right\|_{L^{2}(D)}+h^{\alpha / 2-1+\beta}\left\|u(t)-u_{h}(t)\right\|_{\widetilde{H}^{\alpha / 2}(D)} \leq C h^{\alpha-2+2 \beta} t^{-1}\|v\|_{L^{2}(D)} .
$$

Proof. Note the error $e(t):=u(t)-u_{h}(t)$ can be represented as

$$
e(t)=\frac{1}{2 \pi \mathrm{i}} \int_{\Gamma} e^{-z t}\left(w-w_{h}\right) d z
$$

where the contour $\Gamma=\left\{z: z=\rho e^{ \pm \mathrm{i} \delta_{1}}, \rho \geq 0\right\}$, and $w=R(z ; A) v$ and $w_{h}=R\left(z ; A_{h}\right) P_{h} v$. By Lemma 4.2, we have

$$
\begin{aligned}
\|e(t)\|_{\widetilde{H}^{\alpha / 2}(D)} & \leq C \int_{\Gamma}\left|e^{-z t}\right|\left\|w-w_{h}\right\|_{\widetilde{H}^{\alpha / 2}(D)} d z \\
& \leq C h^{\alpha / 2-1+\beta}\|v\|_{L^{2}(D)} \int_{0}^{\infty} e^{-\rho t \cos \delta_{1}} d \rho \leq C h^{\alpha / 2-1+\beta} t^{-1}\|v\|_{L^{2}(D)} .
\end{aligned}
$$

A similar argument also yields the $L^{2}(D)$-estimate.

4.2. Error estimate for smooth initial data. Next we turn to the case of smooth initial data, i.e., $v \in D(A)$. In order to obtain a uniform bound of the error, we employ an alternative integral representation. With $v_{h}=R_{h} v$, then there holds

$$
\begin{aligned}
u(t)-u_{h}(t) & =\int_{\Gamma} e^{-z t}\left(R(z ; A) v-R\left(z ; A_{h}\right) R_{h} v\right) d z \\
& =\int_{\Gamma_{\delta_{1}}^{t}} e^{-z t}\left(R(z ; A) v-R\left(z ; A_{h}\right) R_{h} v\right) d z
\end{aligned}
$$

where $\Gamma_{\delta_{1}}^{t}=\Gamma_{1} \cup \Gamma_{2} \cup \Gamma_{t}, \Gamma_{1}=\left\{z: z=\rho e^{\mathrm{i} \delta_{1}}, \rho \geq t^{-1}\right\}, \Gamma_{2}=\left\{z: z=\rho e^{-\mathrm{i} \delta_{1}}, \rho \geq t^{-1}\right\}$, and $\Gamma_{t}=\left\{z: z=t^{-1} e^{\mathrm{i} \theta}, \delta_{1} \leq|\theta| \leq \pi\right\}$, cf. Fig. 1(b). Then using the identities

$$
R(z ; A)=A A^{-1} R(z ; A)=A\left(z^{-1} R(z ; A)-z^{-1} A^{-1}\right)=z^{-1} R(z ; A) A-z^{-1} I
$$


and $\int_{\Gamma_{\delta_{1}}^{t}} e^{-s t} z^{-1} d z=0$, the error $u(t)-u_{h}(t)$ can be represented as

$$
u(t)-u_{h}(t)=\int_{\Gamma_{\delta_{1}}^{t}} z^{-1} e^{-z t}\left(w-w_{h}\right) d z,
$$

where $w=R(z ; A) A v$ and $w_{h}=R\left(z ; A_{h}\right) A_{h} R_{h} v$.

Lemma 4.3. For any $\varphi \in \widetilde{H}^{\alpha / 2}(D)$ and $z \in \Gamma_{\delta_{1}}^{t}$, there holds

$$
|z|\|\varphi\|_{L^{2}(D)}^{2}+\|\varphi\|_{\widetilde{H}^{\alpha / 2}(D)}^{2} \leq C\left|z\|\varphi\|_{L^{2}(D)}^{2}-A(\varphi, \varphi)\right| .
$$

Proof. Note that $\Gamma_{1} \cup \Gamma_{2} \subset \Gamma$, thus it suffices to consider $\Gamma_{t}$. Set $z_{t}=t^{-1} e^{\mathrm{i} \delta_{1}}$, then it is obvious that for $z \in \Gamma_{t}$ and $\varphi \in \widetilde{H}^{\alpha / 2}(D)$ we have $\operatorname{dist}\left(z, \Sigma_{\varphi}\right) \geq \operatorname{dist}\left(z_{t}, \Sigma_{\varphi}\right)$, cf. Fig. 1(b). Thus the argument in proving (4.2) yields the desired result.

Remark 4.1. For $v \in L^{2}(D), z \in \Gamma_{t}$, let $w=R(z ; A) v$ and $w_{h}=R\left(z ; A_{h}\right) P_{h} v$. Then the argument in Lemma 4.2 and Lemma 4.3 yield the estimate (4.3).

Theorem 4.2. Let $u$ and $u_{h}$ be solutions of problem (3.1) and (4.1) with $v \in D(A)$ and $v_{h}=R_{h} v$, respectively. Then for any $\beta \in[0,1 / 2)$, there holds

$$
\left\|u(t)-u_{h}(t)\right\|_{L^{2}(D)}+h^{\alpha / 2-1+\beta}\left\|u(t)-u_{h}(t)\right\|_{\widetilde{H}^{\alpha / 2}(D)} \leq C h^{\alpha-2+2 \beta}\|A v\|_{L^{2}(D)} .
$$

Proof. Let $w=R(z ; A) A v$ and $w_{h}=R\left(z ; A_{h}\right) A_{h} R_{h} v$. Together with the identity $A_{h} R_{h}=$ $P_{h} A$, Remark 4.1 gives

$$
\left\|w_{h}-w\right\|_{L^{2}(D)}+h^{\alpha / 2-1+\beta}\left\|w_{h}-w\right\|_{\widetilde{H}^{\alpha / 2}(D)} \leq C h^{\alpha-2+2 \beta}\|A v\|_{L^{2}(D)} .
$$

Now it follows from this and the representation (4.8) that

$$
\begin{aligned}
\left\|u(t)-u_{h}(t)\right\|_{\widetilde{H}^{\alpha / 2}(D)} & \leq C \int_{\Gamma_{\delta_{1}}^{t}}\left|z^{-1}\left\|e^{-z t} \mid\right\| w-w_{h} \|_{\widetilde{H}^{\alpha / 2}(D)} d z\right. \\
& \leq C h^{\alpha / 2-1+\beta}\|A v\|_{L^{2}(D)} \int_{\Gamma_{\delta_{1}}^{t}}\left|z^{-1}\right|\left|e^{-z t}\right| d z .
\end{aligned}
$$

It suffices to bound the integral term. First we note that

$$
\int_{\Gamma_{1}}\left|z^{-1}\right|\left|e^{-z t}\right| d z=\int_{t^{-1}}^{\infty} \rho^{-1} e^{-\rho t \cos \delta_{1}} d \rho \leq \int_{\cos \delta_{1}}^{\infty} x^{-1} e^{-x} d x \leq C,
$$

which is also valid for the integral on the curve $\Gamma_{2}$. Further, we have

$$
\int_{\Gamma_{t}}\left|z^{-1}\right|\left|e^{-z t}\right| d z=\int_{\delta_{1}}^{2 \pi-\delta_{1}} e^{\cos \theta} d \theta \leq C .
$$

Hence we obtain the $\widetilde{H}^{\alpha / 2}(D)$-estimate. The $L^{2}(D)$-estimate follows analogously.

\section{ERROR ANALYSIS FOR FULLY DISCRETE SCHEME}

Now we turn to error estimates for fully discrete schemes, obtained with either the backward Euler method or the Crank-Nicolson method in time.

5.1. Backward Euler method. We first consider the backward Euler method for approximating the first-order time derivative: for $n=1,2, \ldots, N$

$$
U^{n}-U^{n-1}+\tau A_{h} U^{n}=0
$$

with $U^{0}=v_{h}$ which is an approximation of the initial data $v$. Consequently

$$
U^{n}=\left(I+\tau A_{h}\right)^{-n} v_{h}, \quad U^{0}=v_{h}, \quad n=1,2, \ldots, N .
$$

By the standard energy method, the backward Euler method is unconditionally stable, i.e., for any $n \in \mathbb{N},\left\|\left(I+\tau A_{h}\right)^{-n}\right\| \leq 1$.

To analyze the scheme (5.1), we need the following smoothing property [5]. 
Lemma 5.1. For $n \in \mathbb{N}, n \geq \gamma>0$ and $s>0$, there exists a constant $C>0$, depending on $\gamma$ only, such that

$$
\left\|A_{h}^{\gamma}\left(I+s A_{h}\right)^{-n}\right\| \leq C n^{-\gamma} s^{-\gamma} .
$$

Proof. Let $r(z)=\frac{1}{1+z}$. Then by [18, Lemma 9.2], for an arbitrary $R>0$ and $\theta \in\left(0, \frac{\pi}{2}\right)$, there exist constants $c, C>0$ and $\epsilon \in(0,1)$ such that

$$
|r(z)| \leq \begin{cases}e^{C|z|}, & \forall|z| \leq \epsilon, \\ e^{-c|z|}, & \forall|z| \leq R,|\arg z| \leq \theta .\end{cases}
$$

Clearly, (5.2) is equivalent to $\left\|\left(n s A_{h}\right)^{\gamma} r\left(s A_{h}\right)^{n}\right\| \leq C$. The fact that $A_{h}$ is sectorial implies that $s A_{h}, s>0$, is also sectorial on $X_{h}$. Hence it suffices to show

$$
\left\|\left(n A_{h}\right)^{\gamma} r\left(A_{h}\right)^{n}\right\| \leq C
$$

Let $F_{n}(z)=(n z)^{\gamma} r(z)^{n}$. Since $r(\infty)=0$, by Lemma 3.3 and Remark 3.1

$$
F_{n}\left(A_{h}\right)=\frac{1}{2 \pi \mathrm{i}} \int_{\Gamma_{\epsilon / n} \cup \Gamma_{\epsilon / n}^{n R} \cup \Gamma^{n R}} F_{n}(z) R\left(z ; A_{h}\right) d z .
$$

First, by (5.3), we deduce that for $z \in \Gamma_{\epsilon / n}$

$$
\left|F_{n}(z)\right| \leq(n|z|)^{\gamma} e^{c n|z|}=\epsilon^{\gamma} e^{c \epsilon} \leq C
$$

Thus we have

$$
\left\|\frac{1}{2 \pi \mathrm{i}} \int_{\Gamma_{\epsilon / n}} F_{n}(z) R\left(z ; A_{h}\right) d z\right\| \leq C \frac{\epsilon}{n} \sup _{z \in \Gamma_{\epsilon / n}}\left\|R\left(z ; A_{h}\right)\right\| \leq C .
$$

Next, we note

$$
\begin{gathered}
\left\|\frac{1}{2 \pi \mathrm{i}} \int_{\substack{\Gamma_{\epsilon / n}^{n R} \\
n R}} F_{n}(z) R\left(z ; A_{h}\right) d z\right\| \leq C \int_{\epsilon / n}^{n R}(n \varrho)^{\gamma} e^{-c n \varrho} \varrho^{-1} d \varrho \\
\leq C \int_{\epsilon}^{n^{2} R} \rho^{\gamma-1} e^{-\rho} d \rho \leq C \int_{0}^{\infty} \rho^{\gamma-1} e^{-\rho} d \rho \leq C .
\end{gathered}
$$

Last, there holds $|1+n z|^{-1} \leq C(n|z|)^{-1}$ for $|z| \geq 1$. Hence for $z \in \Gamma^{n R}$,

$$
\left|F_{n}(z)\right| \leq C n^{2 \gamma-n} R^{\gamma-n} \leq C, \quad \forall n \geq \gamma
$$

Thus we have the following bound for the integral on the curve $\Gamma^{n R}$ :

$$
\left\|\frac{1}{2 \pi \mathrm{i}} \int_{\Gamma^{n R}} F_{n}(z) R\left(z ; A_{h}\right) d z\right\| \leq C n R \sup _{z \in \Gamma^{n} R}\left\|R\left(z ; A_{h}\right)\right\| \leq C .
$$

This completes the proof of the lemma.

Now we derive an error estimate for the fully discrete scheme (5.1) in case of smooth initial data, i.e., $v \in D(A)$.

Theorem 5.1. Let $u$ and $U^{n}$ be solutions of problem (3.1) and (5.1) with $v \in D(A)$ and $U^{0}=R_{h} v$, respectively. Then for $t_{n}=n \tau$ and any $\beta \in[0,1 / 2)$, there holds

$$
\left\|u\left(t_{n}\right)-U^{n}\right\|_{L^{2}(D)} \leq C\left(h^{\alpha-2+2 \beta}+\tau\right)\|A v\|_{L^{2}(D)} .
$$

Proof. Note that the error $e^{n}=u\left(t_{n}\right)-U^{n}$ can be split into

$$
e^{n}=\left(u\left(t_{n}\right)-u_{h}\left(t_{n}\right)\right)+\left(u_{h}\left(t_{n}\right)-U^{n}\right):=\widetilde{\varrho}^{n}+\widetilde{\vartheta}^{n},
$$

where $u_{h}$ denotes the semidiscrete Galerkin solution with $v_{h}=R_{h} v$. By Theorem 4.2, the term $\widetilde{\varrho}^{n}$ satisfies the following estimate

$$
\left\|\widetilde{\varrho}^{n}\right\|_{L^{2}(D)} \leq C h^{\alpha-2+2 \beta}\|A v\|_{L^{2}(D)} .
$$


Next we bound the term $\widetilde{\vartheta}^{n}$. Note that for $n \geq 1$,

$$
\begin{aligned}
\widetilde{\vartheta}^{n} & =E_{h}(n \tau)-\left(I+\tau A_{h}\right)^{-n} v_{h} \\
& =-\int_{0}^{\tau} \frac{d}{d s}\left(E_{h}(n(\tau-s))\left(I+s A_{h}\right)^{-n} v_{h}\right) d s \\
& =-\int_{0}^{\tau} n s A_{h}^{2} E_{h}(n(\tau-s))\left(I+s A_{h}\right)^{-n-1} v_{h} d s .
\end{aligned}
$$

Then by Lemmas 3.2 and 5.1 we have

$$
\begin{aligned}
\left\|\widetilde{\vartheta}^{n}\right\|_{L^{2}(D)} & \leq C n^{1 / 2} \int_{0}^{\tau} s(\tau-s)^{-1 / 2}\left\|A_{h}^{3 / 2}\left(I+s A_{h}\right)^{-n-1} R_{h} v\right\|_{L^{2}(D)} d s \\
& \leq C n^{1 / 2} \int_{0}^{\tau} s^{1 / 2}(n+1)^{-1 / 2}(\tau-s)^{-1 / 2}\left\|A_{h} R_{h} v\right\|_{L^{2}(D)} d s \\
& \leq C \tau\left\|A_{h} R_{h} v\right\|_{L^{2}(D)} .
\end{aligned}
$$

The desired result follows from the identity $A_{h} R_{h}=P_{h} A$ and the $L^{2}(D)$-stability of the projection $P_{h}$.

Next we give an error estimate for $L^{2}(D)$ initial data $v$.

Theorem 5.2. Let $u$ and $U^{n}$ be solutions of problem (3.1) and (5.1) with $v \in L^{2}(D)$ and $U^{0}=P_{h} v$, respectively. Then for $t_{n}=n \tau$ and any $\beta \in[0,1 / 2)$, there holds

$$
\left\|u\left(t_{n}\right)-U^{n}\right\|_{L^{2}(D)} \leq C\left(h^{\alpha-2+2 \beta}+\tau\right) t_{n}^{-1}\|v\|_{L^{2}(D)} .
$$

Proof. Like before, we split the error $e^{n}=u\left(t_{n}\right)-U^{n}$ into

$$
e^{n}=\left(u\left(t_{n}\right)-u_{h}\left(t_{n}\right)\right)+\left(u_{h}\left(t_{n}\right)-U^{n}\right):=\widetilde{\varrho}^{n}+\widetilde{\vartheta}^{n},
$$

where $u_{h}$ denotes the semidiscrete Galerkin solution with $v_{h}=P_{h} v$. In view of Theorem 4.1 it remains to estimate the term $\widetilde{\vartheta}^{n}$. By identity (5.4) and Lemmas 5.1 and 3.2, we have for $n \geq 1$

$$
\begin{aligned}
\left\|\widetilde{\vartheta}^{n}\right\|_{L^{2}(D)} & \leq C n \int_{0}^{\tau} s\left\|A_{h}^{3 / 2}\left(I+s A_{h}\right)^{-n-1} A_{h}^{1 / 2} E_{h}(n(\tau-s)) P_{h} v\right\|_{L^{2}(D)} d s \\
& \leq C n \int_{0}^{\tau} s s^{-3 / 2}(n+1)^{-3 / 2}\left\|A_{h}^{1 / 2} E_{h}(n(\tau-s)) P_{h} v\right\|_{L^{2}(D)} d s \\
& \leq C n^{-1 / 2} \int_{0}^{\tau} s^{-1 / 2} n^{-1 / 2}(\tau-s)^{-1 / 2}\left\|P_{h} v\right\|_{L^{2}(D)} d s \leq C \tau t_{n}^{-1}\|v\|_{L^{2}(D)} .
\end{aligned}
$$

This completes the proof of the theorem.

5.2. Crank-Nicolson method. Now we turn to the fully discrete scheme based on the Crank-Nicolson method. It reads

$$
U^{n}-U^{n-1}+\tau A_{h} U^{n-1 / 2}=0, \quad U^{0}=v_{h}, \quad n=1,2, \ldots, N,
$$

where $U^{n-1 / 2}=\frac{1}{2}\left(U^{n}+U^{n-1}\right)$. Therefor we have

$$
U^{n}=\left(I+\frac{\tau}{2} A_{h}\right)^{-n}\left(I-\frac{\tau}{2} A_{h}\right)^{n} v_{h}, \quad n=1,2, \ldots, N .
$$

It can be verified by the energy method that the Crank-Nicolson method is unconditionally stable, i.e., for any $n \in \mathbb{N},\left\|\left(I+\frac{\tau}{2} A_{h}\right)^{-n}\left(I-\frac{\tau}{2} A_{h}\right)^{n}\right\| \leq 1$.

For the error analysis, we need a result on the rational function

$$
r_{c n}(z)=\frac{1-\frac{z}{2}}{1+\frac{z}{2}}
$$

Lemma 5.2. For any arbitrary $R>0$, there exist $C>0$ and $c>0$ such that

$$
\left|e^{-n z}-r_{c n}(z)^{n}\right| \leq \begin{cases}C e^{-\frac{c n}{|z|}}, & |\arg z| \leq \delta_{1},|z| \geq R \\ C n|z|^{3} e^{-c n|z|}, & |\arg z| \leq \delta_{1},|z| \leq R\end{cases}
$$


Proof. The proof of general cases can be found in [18, Lemmas 9.2 and 9.4]. We briefly sketch the proof here. By setting $w=1 / z$, the first inequality follows from

$$
r_{c n}(z)=\frac{1-\frac{z}{2}}{1+\frac{z}{2}}=-\frac{1-2 w}{1+2 w}=-r(4 w)=-e^{-4 w+O\left(w^{2}\right)}, \quad w \rightarrow 0,
$$

and that for $c \leq \cos \delta_{1}$,

$$
\left|e^{-z}\right|=e^{-\Re z} \leq e^{-c|z|} \leq C e^{-\frac{c}{|z|}},|\arg z| \leq \delta_{1},|z| \geq R
$$

The first estimate now follows by the triangle inequality. Meanwhile, we observe that

$$
\begin{aligned}
& \left|r_{c n}(z)-e^{-z}\right| \leq C|z|^{3}, \quad|z| \leq R,|\arg z| \leq \delta_{1}, \\
& \left|r_{c n}(z)\right| \leq e^{-c|z|}, \quad|\arg z| \leq \delta_{1},|z| \leq R .
\end{aligned}
$$

Consequently for $z$ under consideration

$$
\left|e^{-n z}-r_{c n}(z)^{n}\right|=\left|\left(e^{-z}-r_{c n}(z)\right) \sum_{j=0}^{n-1} r_{c n}(z)^{j} e^{-(n-1-j) z}\right| \leq C|z|^{3} n e^{-c n|z|} .
$$

This completes the proof of the lemma.

Now we can state an $L^{2}(D)$-norm estimate for (5.6) in case of smooth initial data.

Theorem 5.3. Let $u$ and $U^{n}$ be solutions of problem (3.1) and (5.6) with $v \in D(A)$ and $U^{0}=R_{h} v$, respectively. Then for $t_{n}=n \tau$ and any $\beta \in[0,1 / 2)$, there holds

$$
\left\|u\left(t_{n}\right)-U^{n}\right\|_{L^{2}(D)} \leq C\left(h^{\alpha-2+2 \beta}+\tau^{2} t_{n}^{-1}\right)\|A v\|_{L^{2}(D)} .
$$

Proof. Like before, we split the error $e^{n}$ into

$$
e^{n}=\left(u\left(t_{n}\right)-u_{h}\left(t_{n}\right)\right)+\left(u_{h}\left(t_{n}\right)-U^{n}\right):=\widetilde{\varrho}^{n}+\widetilde{\vartheta}^{n},
$$

where $u_{h}$ denotes the semidiscrete Galerkin solution with $v_{h}=R_{h} v$. Then by Theorem 4.2 the term $\widetilde{\varrho}^{n}$ satisfies the following estimate

$$
\left\|\widetilde{\varrho}^{n}\right\|_{L^{2}(D)} \leq C h^{\alpha-2+2 \beta}\|A v\|_{L^{2}(D)} .
$$

It remains to bound $\widetilde{\vartheta}^{n}=E_{h}(n \tau) v_{h}-r_{c n}\left(\tau A_{h}\right)^{n} v_{h}$ by

$$
\left\|\widetilde{\vartheta}^{n}\right\|_{L^{2}(D)} \leq C \tau^{2} t_{n}^{-1}\left\|A_{h} v_{h}\right\|_{L^{2}(D)} .
$$

Note that $\tau A_{h}$ is also sectorial with the same constant as $A_{h}$, and further

$$
\left\|\left(z I-\tau A_{h}\right)^{-1}\right\|=\tau^{-1}\left\|\frac{z}{\tau}-A_{h}\right\| \leq C \frac{1}{|z|} .
$$

With $t_{n}=n \tau$, it suffices to show

$$
\left\|A_{h}^{-1}\left(E_{h}(n)-r_{c n}\left(A_{h}\right)^{n}\right)\right\| \leq C n^{-1}
$$

By Lemma 3.3, there holds

$$
A_{h}^{-1} r_{c n}\left(A_{h}\right)^{n}=\frac{1}{2 \pi \mathrm{i}} \int_{\Gamma_{\epsilon} \cup \Gamma_{\epsilon}^{R} \cup \Gamma^{R}} r_{c n}(z)^{n} z^{-1} R\left(z ; A_{h}\right) d z .
$$

Since $\left\|r_{c n}(z)^{n} z^{-1} R\left(z ; A_{h}\right)\right\|=O\left(z^{-2}\right)$ for large $z$, we can let $R$ tend to $\infty$. Further, by [18. Lemma 9.3], we have

$$
A_{h}^{-1} E_{h}(n)=\frac{1}{2 \pi \mathrm{i}} \int_{\Gamma_{\epsilon} \cup \Gamma_{\epsilon}^{\infty}} e^{-n z} z^{-1} R\left(z ; A_{h}\right) d z .
$$

By Lemma 5.2 .

$$
\left\|\left(e^{-n z}-r_{c n}(z)^{n}\right) z^{-1} R\left(z ; A_{h}\right)\right\|=O(z) \quad \text { as } z \rightarrow 0,|\arg z| \leq \delta_{1},
$$

and consequently, by taking $\epsilon \rightarrow 0$, there holds

$$
A_{h}^{-1}\left(E_{h}(n)-r_{c n}\left(A_{h}\right)^{n}\right)=\frac{1}{2 \pi \mathrm{i}} \int_{\Gamma}\left(e^{-n z}-r_{c n}(z)^{n}\right) z^{-1} R\left(z ; A_{h}\right) d z,
$$


where the sector $\Gamma$ is given by $\Gamma=\left\{z: z=\rho e^{ \pm \mathrm{i} \delta_{1}}, \rho \geq 0\right\}$. By applying Lemma 5.2 with $R=1$, we deduce

$$
\begin{aligned}
\left\|A_{h}^{-1}\left(E_{h}(n)-r_{c n}\left(A_{h}\right)^{n}\right)\right\| & =\left\|\frac{1}{2 \pi \mathrm{i}} \int_{\Gamma}\left(e^{-n z}-r_{c n}(z)^{n}\right) z^{-1} R\left(z ; A_{h}\right) d z\right\| \\
& \leq C \int_{0}^{1} \rho n e^{-c n \rho} d \rho+C \int_{1}^{\infty} \rho^{-2} e^{-c n \rho^{-1}} d \rho \\
& \leq C n^{-1}\left(\int_{0}^{\infty} \varrho e^{-\varrho} d \varrho+\int_{0}^{\infty} e^{-\varrho} d \varrho\right) \leq C n^{-1} .
\end{aligned}
$$

This completes the proof of the theorem.

Now we turn to the case of nonsmooth initial data, i.e., $v \in L^{2}(D)$. It is known that in case of the standard parabolic equation, the Crank-Nicolson method fails to give an optimal error estimate for such data unconditionally because of a lack of smoothing property [13, 19. Hence we employ a damped Crank-Nicolson scheme, which is realized by replacing the first two time steps by the backward Euler method. Further, we denote

$$
r_{d c n}(z)^{n}=r_{b w}(z)^{2} r_{c n}(z)^{n-2} .
$$

The damped Crank-Nicolson scheme is also unconditionally stable. Further, the function $r_{d c n}(z)$ has the following estimates [7, Lemma 2.2].

Lemma 5.3. Let $r_{d c n}$ be defined as in (5.8) then there exist positive constants $\epsilon, R, C, c$ such that

$$
\begin{aligned}
& \left|r_{d c n}(z)^{n}\right| \leq \begin{cases}(1+C|z|)^{n}, & |z|<\epsilon \\
e^{-c n|z|}, & \forall|z| \leq 1,|\arg (z)| \leq \delta_{1} \\
C|z|^{-2} e^{-\frac{c(n-2)}{|z|}}, & \forall|z| \geq 1,|\arg (z)| \leq \delta_{1}, n \geq 2 ; \\
C|z|^{-2}, & |z| \geq R, n \geq 2\end{cases} \\
& \left|r_{b w}(z)^{2}-e^{-2 z}\right| \leq C|z|^{2}, \quad \forall|z| \leq \epsilon \quad \text { or } \quad|\arg (z)| \leq \delta_{1} .
\end{aligned}
$$

Theorem 5.4. Let $u$ be the solution of problem (3.1), and $U^{n}=r_{d c n}\left(\tau A_{h}\right) U^{0}$ with $v \in L^{2}(D)$ and $U^{0}=P_{h} v$. Then for $t_{n}=n \tau$ and any $\beta \in[0,1 / 2)$, there holds

$$
\left\|u\left(t_{n}\right)-U^{n}\right\|_{L^{2}(D)} \leq C\left(h^{\alpha-2+2 \beta} t_{n}^{-1}+\tau^{2} t_{n}^{-2}\right)\|v\|_{L^{2}(D)} .
$$

Proof. We split the error $e^{n}=u\left(t_{n}\right)-U^{n}$ as (5.5). Since the bound on $\widetilde{\varrho}^{n}$ follows from Theorem 4.1 it remains to bound $\widetilde{\vartheta}^{n}=E_{h}(\tau n) v_{h}-r_{d c n}\left(\tau A_{h}\right)^{n} v_{h}$ for $n \geq 1$ as

$$
\left\|\widetilde{\vartheta}^{n}\right\|_{L^{2}(D)} \leq C \tau^{2} t_{n}^{-2}\left\|v_{h}\right\|_{L^{2}(D)} .
$$

Let $F_{n}(z)=e^{-n z}-r_{d c n}(z)^{n}$. Then it suffices to show for $n \geq 1$

$$
\left\|F_{n}\left(A_{h}\right)\right\| \leq C n^{-2} \text {. }
$$

The estimate is trivial for $n=1,2$ by boundedness. For $n>2$, we split $F_{n}(z)$ into

$$
\begin{aligned}
F_{n}(z) & =r_{b w}(z)^{2}\left(e^{-(n-2) z}-r_{c n}(z)^{n-2}\right)+e^{-(n-2) z}\left(e^{-2 z}-r_{b w}(z)^{2}\right) \\
& :=f_{1}(z)+f_{2}(z) .
\end{aligned}
$$

It follows from 18. Lemma 9.1 and Lemma 9.3] that

$$
\begin{aligned}
r_{d c n}\left(A_{h}\right)^{n} & =\frac{1}{2 \pi \mathrm{i}} \int_{\Gamma_{\epsilon} \cup \Gamma_{\epsilon}^{R} \cup \Gamma^{R}} r_{d c n}(z)^{n} R\left(z ; A_{h}\right) d z, \\
E_{h}(n) & =\frac{1}{2 \pi \mathrm{i}} \int_{\Gamma_{\epsilon} \cup \Gamma_{\epsilon}^{\infty}} e^{-n z} R\left(z ; A_{h}\right) d z .
\end{aligned}
$$

Using the fact $\left\|r_{d c n}(z)^{n} R\left(z ; A_{h}\right)\right\|=O\left(z^{-3}\right)$ as $z \rightarrow \infty$, we may let $R \rightarrow \infty$ to obtain

$$
F_{n}\left(A_{h}\right)=\frac{1}{2 \pi \mathrm{i}} \int_{\Gamma_{\epsilon} \cup \Gamma_{\epsilon}^{\infty}} F_{n}(z) R\left(z ; A_{h}\right) d z .
$$


Further, by Lemma 5.3. $\left\|F_{n}(z) R\left(z ; A_{h}\right)\right\|=O(z)$ as $z \rightarrow 0$, and consequently by taking $\epsilon \rightarrow 0$ and setting $\Gamma=\left\{z: z=\rho e^{ \pm i \delta_{1}}, \rho \geq 0\right\}$, we have

$$
\begin{aligned}
F_{n}\left(A_{h}\right) & =\frac{1}{2 \pi \mathrm{i}} \int_{\Gamma} F_{n}(z) R\left(z ; A_{h}\right) d z \\
& =\frac{1}{2 \pi \mathrm{i}} \int_{\Gamma}\left(f_{1}(z)+f_{2}(z)\right) R\left(z ; A_{h}\right) d z .
\end{aligned}
$$

Now we estimate the two terms separately. First, by Lemmas 5.2 and 5.3 we get

$$
\begin{aligned}
& \left|f_{1}(z)\right| \leq\left|r_{d c n}(z)^{n}\right|+\left|r_{b w}(z)^{2}\right|\left|e^{-(n-2) z}\right| \leq C|z|^{-2} e^{-\frac{c n}{|z|}}, \quad z \in \Gamma,|z| \geq 1, \\
& \left|f_{1}(z)\right| \leq\left|r_{b w}(z)^{2}\right|\left|r_{c n}(z)^{n-2}-e^{-(n-2) z}\right| \leq C|z|^{3} n e^{-c n|z|}, \quad z \in \Gamma,|z| \leq 1 .
\end{aligned}
$$

Repeating the argument for (5.7) gives that for $n>2$

$$
\left\|\frac{1}{2 \pi \mathrm{i}} \int_{\Gamma} f_{1}(z) R\left(z ; A_{h}\right) d z\right\| \leq C n^{-2}
$$

As to other term, we deduce from (5.9) that

$$
\left|f_{2}(z)\right| \leq\left|e^{-(n-2) z}\right|\left|r_{b w}(z)^{2}-e^{-2 z}\right| \leq C|z|^{2}, \quad \forall|z| \leq \epsilon,
$$

and thus we can change the integration path $\Gamma$ to $\Gamma_{\epsilon / n}^{\infty} \cup \Gamma_{\epsilon / n}$. Further, we deduce from Lemma 5.3 that

$$
\left|f_{2}(z)\right|=\left|e^{-(n-2) z}\left(r_{b w}(z)^{2}-e^{-2 z}\right)\right| \leq C e^{-c(n-2)|z|}|z|^{2}, \quad \forall z \in \Gamma_{\epsilon / n}^{\infty} .
$$

Thus, we derive the following bound for $n>2$

$$
\left\|\frac{1}{2 \pi \mathrm{i}} \int_{\Gamma} f_{1}(z) R\left(z ; A_{h}\right) d z\right\| \leq C \int_{\epsilon / n}^{\infty} e^{-c(n-2) \rho} \rho d \rho+C \int_{\Gamma_{\epsilon / n}} \rho d \rho \leq C n^{-2} .
$$

This completes the proof of the theorem.

\section{Numerical RESUlts}

In this section, we present numerical experiments to verify our theoretical results. To this end, we consider the following three examples:

(a) smooth initial data: $v(x)=x(x-1)$, which lies in $\widetilde{H}^{3 / 2-\epsilon}(D)$.

(b) nonsmooth initial data: (b1) $v(x)=\chi_{(1 / 2,1)}(x)$, the characteristic function of the interval $(1 / 2,1)$; (b2) $v(x)=x^{1 / 4}$; Note that in (b1) $v \in \widetilde{H}^{1 / 2-\epsilon}(D)$ while in (b2) $v \in \widetilde{H}^{1 / 4-\epsilon}(D)$, for any $\epsilon>0$.

(c) discontinuous potential $q(x)=\chi_{(0,1 / 2)}(x)$.

We examine separately the spatial and temporal convergence rates at $t=1$. For the case of nonsmooth initial data, we are especially interested in the errors for $t$ close to zero, and thus we also present the errors at $t=0.1,0.01,0.005$, and 0.001 . The exact solutions to these examples are not available in closed form, and hence we compute the reference solution on a very refined mesh. We measure the accuracy of the numerical approximation $U^{n}$ by the normalized errors $\left\|u\left(t_{n}\right)-U^{n}\right\|_{L^{2}(D)} /\|v\|_{L^{2}(D)}$ and $\| u\left(t_{n}\right)-$ $U^{n}\left\|_{\widetilde{H}^{\alpha / 2}(D)} /\right\| v \|_{L^{2}(D)}$. The normalization enables us to observe the behavior of the errors with respect to time in case of nonsmooth initial data. To study the rate of convergence in space, we use a time step size $\tau=10^{-5}$ so that the time discretization error is negligible, and we have the space discretization error only. 
6.1. Numerical results for example (a): smooth initial data. In Table 1 we show the errors $\left\|u\left(t_{n}\right)-U^{n}\right\|_{L^{2}(D)}$ and $\left\|u\left(t_{n}\right)-U^{n}\right\|_{\widetilde{H}^{\alpha / 2}(D)}$ with the backward Euler method. We have set $\tau=10^{-5}$, so that the error incurred by temporal discretization is negligible. In the table, ratio refers to the ratio of the errors when the mesh size $h$ (or time step size $\tau$ ) halves, and the numbers in the bracket denote theoretical convergence rates. The numerical results show $O\left(h^{\alpha-1 / 2}\right)$ and $O\left(h^{\alpha / 2-1 / 2}\right)$ convergence rates for the $L^{2}(D)$ - and $\widetilde{H}^{\alpha / 2}(D)$-norms of the error, respectively. In Fig. 2] we plot the results for $\alpha=1.5$ at $t=1$ in a log-log scale. The $\widetilde{H}^{\alpha / 2}(D)$-norm estimate is fully confirmed, but the $L^{2}(D)$ norm estimate is suboptimal: the empirical convergence rate is one half order higher than the theoretical one. The suboptimality is attributed to the low regularity of the adjoint solution, used in Nitsche's trick. In view of the singularity of the term $x^{\alpha-1}$ in the solution representation, cf. Remark 2.1 the spatial discretization error is concentrated around the origin.

TABLE 1. $L^{2}$ - and $\tilde{H}^{\alpha / 2}$-norms of the error for example (a), smooth initial data, with $\alpha=1.25,1.5,1.75$ for backward Euler method and $\tau=10^{-5}$; in the last column in brackets is the theoretical rate.

\begin{tabular}{|c|c|cccccc|c|}
\hline$\alpha$ & $h$ & $1 / 16$ & $1 / 32$ & $1 / 64$ & $1 / 128$ & $1 / 256$ & $1 / 512$ & ratio \\
\hline 1.25 & $L^{2}$ & $5.13 \mathrm{e}-3$ & $2.89 \mathrm{e}-3$ & $1.69 \mathrm{e}-3$ & $1.00 \mathrm{e}-3$ & $6.03 \mathrm{e}-4$ & $3.71 \mathrm{e}-4$ & $\approx 0.76(0.25)$ \\
\cline { 2 - 7 } & $H^{\alpha / 2}$ & $4.93 \mathrm{e}-2$ & $4.39 \mathrm{e}-2$ & $3.98 \mathrm{e}-2$ & $3.62 \mathrm{e}-2$ & $3.29 \mathrm{e}-2$ & $3.00 \mathrm{e}-2$ & $\approx 0.14(0.13)$ \\
\hline \multirow{2}{*}{1.5} & $L^{2}$ & $3.62 \mathrm{e}-4$ & $1.70 \mathrm{e}-4$ & $8.37 \mathrm{e}-5$ & $4.17 \mathrm{e}-5$ & $2.09 \mathrm{e}-5$ & $1.06 \mathrm{e}-5$ & $\approx 1.02(0.50)$ \\
\cline { 2 - 7 } & $H^{\alpha / 2}$ & $7.57 \mathrm{e}-3$ & $6.25 \mathrm{e}-3$ & $5.20 \mathrm{e}-3$ & $4.33 \mathrm{e}-3$ & $3.58 \mathrm{e}-3$ & $2.91 \mathrm{e}-3$ & $\approx 0.27(0.25)$ \\
\hline \multirow{2}{*}{1.75} & $L^{2}$ & $1.12 \mathrm{e}-5$ & $4.61 \mathrm{e}-6$ & $1.92 \mathrm{e}-6$ & $8.02 \mathrm{e}-7$ & $3.35 \mathrm{e}-7$ & $1.37 \mathrm{e}-7$ & $\approx 1.26(0.75)$ \\
\cline { 2 - 6 } & $H^{\alpha / 2}$ & $4.63 \mathrm{e}-4$ & $3.47 \mathrm{e}-4$ & $2.58 \mathrm{e}-4$ & $1.95 \mathrm{e}-4$ & $1.46 \mathrm{e}-4$ & $1.06 \mathrm{e}-4$ & $\approx 0.42(0.38)$ \\
\hline
\end{tabular}

In Table 2 we let the spacial step size $h \rightarrow 0$ and examine the temporal convergence order, and observe an $O(\tau)$ and $O\left(\tau^{2}\right)$ convergence rates for the backward Euler method and Crank-Nicolson method, respectively. Note that for the case $\alpha=1.75$, the CrankNicolson method fails to achieve an optimal convergence order. This is attributed to the fact that $v$ is not in the domain of the differential operator ${ }_{0}^{R} D_{x}^{\alpha}$ for $\alpha>1.5$. In contrast, the damped Crank-Nicolson method yields the desired $O\left(\tau^{2}\right)$ convergence rates, cf. Table 3 This confirms the discussions in Section 5.2

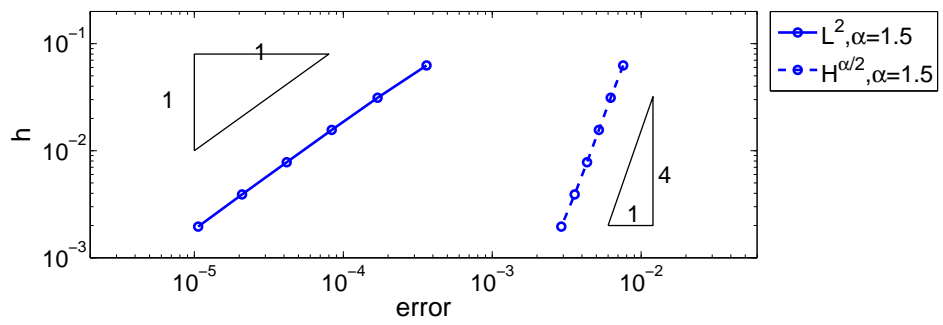

FiguRE 2. Numerical results for example (a) (smooth data) with $\alpha=$ 1.5 at $t=1$.

6.2. Numerical results for nonsmooth initial data: example (b). In Tables 4 5 and 6] we present numerical results for problem (b1). Table 4 shows that the spatial convergence rate is of the order $O\left(h^{\alpha-1+\beta}\right)$ in $L^{2}(D)$-norm and $O\left(h^{\alpha / 2-1+\beta}\right)$ in $\widetilde{H}^{\alpha / 2}(D)$, whereas Table 5 shows that the temporal convergence order is of order $O(\tau)$ and $O\left(\tau^{2}\right)$ for the backward Euler method and damped Crank-Nicolson method, respectively. For the case of nonsmooth initial data, we are interested in the errors for $t$ closed to zero, thus we check the error at $t=0.1,0.01,0.005$ and 0.001 . From Table 6 we observe that 
TABLE 2. $L^{2}$-norm of the error for example (a), non-smooth initial data, with $\alpha=1.25,1.5,1.75, h=2 \times 10^{-5}(\mathrm{BE}-$ backward Euler, CN Crank-Nicolson)

\begin{tabular}{|c|c|c|c|c|c|c|c|}
\hline & $\tau$ & $1 / 10$ & $1 / 20$ & $1 / 40$ & $1 / 80$ & $1 / 160$ & ratio \\
\hline \multirow[t]{3}{*}{$\overline{\mathrm{BE}}$} & $\alpha=1.25$ & $3.01 \mathrm{e}-2$ & $1.41 \mathrm{e}-2$ & $6.63 \mathrm{e}-3$ & $3.10 \mathrm{e}-3$ & $1.41 \mathrm{e}-3$ & \multirow{3}{*}{$\begin{array}{l}\approx 1.10(1.00) \\
\approx 1.13(1.00) \\
\approx 1.20(1.00)\end{array}$} \\
\hline & $\alpha=1.5$ & $1.32 \mathrm{e}-2$ & $5.88 \mathrm{e}-3$ & $2.71 \mathrm{e}-3$ & $1.25 \mathrm{e}-3$ & $5.62 \mathrm{e}-4$ & \\
\hline & $\alpha=1.75$ & $4.79 \mathrm{e}-3$ & $1.88 \mathrm{e}-3$ & $7.95 \mathrm{e}-3$ & $3.53 \mathrm{e}-4$ & $1.55 \mathrm{e}-4$ & \\
\hline \multirow[t]{3}{*}{$\mathrm{CN}$} & $\alpha=1.25$ & $3.18 \mathrm{e}-3$ & $5.98 \mathrm{e}-4$ & $1.35 \mathrm{e}-4$ & $3.32 \mathrm{e}-5$ & $8.52 \mathrm{e}-6$ & \multirow{3}{*}{$\begin{array}{l}\approx 2.10(2.00) \\
\approx 2.06(2.00) \\
\approx 1.73(--)\end{array}$} \\
\hline & $\alpha=1.5$ & $3.22 \mathrm{e}-3$ & $7.32 \mathrm{e}-4$ & $1.75 \mathrm{e}-4$ & $4.32 \mathrm{e}-5$ & $1.05 \mathrm{e}-5$ & \\
\hline & $\alpha=1.75$ & $3.67 \mathrm{e}-3$ & $1.09 \mathrm{e}-3$ & $3.33 \mathrm{e}-4$ & $1.08 \mathrm{e}-4$ & $3.09 \mathrm{e}-5$ & \\
\hline
\end{tabular}

TABLE 3. $L^{2}$-norm of the error for example (a), non-smooth initial data, for damped Crank-Nicolson method with $\alpha=1.75$ and $h=2 \times 10^{-5}$.

\begin{tabular}{|c|ccccc|c|}
\hline$\tau$ & $1 / 10$ & $1 / 20$ & $1 / 40$ & $1 / 80$ & $1 / 160$ & ratio \\
\hline$\alpha=1.75$ & $7.57 \mathrm{e}-4$ & $1.98 \mathrm{e}-4$ & $5.45 \mathrm{e}-5$ & $1.40 \mathrm{e}-5$ & $2.90 \mathrm{e}-6$ & $\approx 1.98(2.00)$ \\
\hline
\end{tabular}

both the $L^{2}(D)$-norm and $\widetilde{H}^{\alpha / 2}(D)$-norm of the error exhibit superconvergence, which theoretically remains to be established. Numerically, for this example. one observes that the solution is smoother than in $\widetilde{H}_{L}^{\alpha-1+\beta}(D)$ for small time $t$, cf. Fig. 3

Similarly, the numerical results for problem (b2) are presented in Tables 7,8 and 9 , see also Fig. 4 for a plot of the results in Table 9 It is observed that the convergence is slower than that for problem (b1), due to the lower solution regularity.

TABLE 4. $L^{2}$ - and $\tilde{H}^{\alpha / 2}$-norms of the error for example (b1), nonsmooth initail data, for backward Euler method with $\tau=10^{-5}$.

\begin{tabular}{|c|c|cccccc|c|}
\hline$\alpha$ & $h$ & $1 / 16$ & $1 / 32$ & $1 / 64$ & $1 / 128$ & $1 / 256$ & $1 / 512$ & ratio \\
\hline \multirow{2}{*}{1.25} & $L^{2}$ & $6.65 \mathrm{e}-3$ & $3.75 \mathrm{e}-3$ & $2.18 \mathrm{e}-3$ & $1.29 \mathrm{e}-3$ & $7.78 \mathrm{e}-4$ & $4.77 \mathrm{e}-4$ & $\approx 0.76(0.25)$ \\
\cline { 2 - 7 } & $H^{\alpha / 2}$ & $6.36 \mathrm{e}-2$ & $5.66 \mathrm{e}-2$ & $5.12 \mathrm{e}-2$ & $4.66 \mathrm{e}-2$ & $4.24 \mathrm{e}-2$ & $3.87 \mathrm{e}-2$ & $\approx 0.14(0.13)$ \\
\hline \multirow{2}{*}{1.5} & $L^{2}$ & $3.78 \mathrm{e}-4$ & $1.77 \mathrm{e}-4$ & $8.56 \mathrm{e}-5$ & $4.22 \mathrm{e}-5$ & $2.09 \mathrm{e}-5$ & $1.04 \mathrm{e}-5$ & $\approx 1.03(0.50)$ \\
\cline { 2 - 7 } & $H^{\alpha / 2}$ & $7.31 \mathrm{e}-3$ & $6.01 \mathrm{e}-3$ & $5.00 \mathrm{e}-3$ & $4.16 \mathrm{e}-3$ & $3.43 \mathrm{e}-3$ & $2.79 \mathrm{e}-3$ & $\approx 0.27(0.25)$ \\
\hline \multirow{2}{*}{1.75} & $L^{2}$ & $2.11 \mathrm{e}-5$ & $9.49 \mathrm{e}-6$ & $4.06 \mathrm{e}-6$ & $1.69 \mathrm{e}-6$ & $6.83 \mathrm{e}-7$ & $2.59 \mathrm{e}-7$ & $\approx 1.27(0.75)$ \\
\cline { 2 - 6 } & $H^{\alpha / 2}$ & $3.63 \mathrm{e}-4$ & $2.69 \mathrm{e}-4$ & $1.99 \mathrm{e}-4$ & $1.50 \mathrm{e}-4$ & $1.12 \mathrm{e}-4$ & $8.19 \mathrm{e}-5$ & $\approx 0.43(0.38)$ \\
\hline
\end{tabular}

TABLE 5. $L^{2}$-norm of the error for example (b1), non-smooth initial data, with $h=2 \times 10^{-5}$ (BE - backward Euler, CN - Crank-Nicolson)

\begin{tabular}{|c|c|c|c|c|c|c|c|}
\hline & $\tau$ & $1 / 10$ & $1 / 20$ & $1 / 40$ & $1 / 80$ & $1 / 160$ & ratio \\
\hline \multirow[t]{3}{*}{$\overline{\mathrm{BE}}$} & $\alpha=1.25$ & $3.73 \mathrm{e}-2$ & $1.80 \mathrm{e}-2$ & $8.53 \mathrm{e}-3$ & $4.00 \mathrm{e}-3$ & $1.81 \mathrm{e}-3$ & $\approx 1.09(1.00)$ \\
\hline & $\alpha=1.5$ & $1.26 \mathrm{e}-2$ & $5.64 \mathrm{e}-3$ & $2.59 \mathrm{e}-3$ & $1.20 \mathrm{e}-3$ & $5.40 \mathrm{e}-4$ & $\approx 1.13(1.00)$ \\
\hline & $\alpha=1.75$ & $3.68 \mathrm{e}-3$ & $1.44 \mathrm{e}-3$ & $6.12 \mathrm{e}-3$ & $2.71 \mathrm{e}-4$ & $1.20 \mathrm{e}-4$ & $\approx 1.19(1.00)$ \\
\hline \multirow[t]{3}{*}{$\mathrm{CN}$} & $\alpha=1.25$ & $3.52 \mathrm{e}-3$ & $9.10 \mathrm{e}-4$ & $2.39 \mathrm{e}-4$ & $5.90 \mathrm{e}-5$ & $1.30 \mathrm{e}-5$ & $\approx 2.01(2.00)$ \\
\hline & $\alpha=1.5$ & $8.86 \mathrm{e}-4$ & $2.42 \mathrm{e}-2$ & $6.46 \mathrm{e}-5$ & $1.61 \mathrm{e}-5$ & $3.44 \mathrm{e}-6$ & $\approx 1.99(2.00)$ \\
\hline & $\alpha=1.75$ & $1.86 \mathrm{e}-4$ & $4.01 \mathrm{e}-5$ & $1.02 \mathrm{e}-5$ & $2.57 \mathrm{e}-6$ & $5.41 \mathrm{e}-7$ & $\approx 2.09(2.00)$ \\
\hline
\end{tabular}

6.3. Numerical results for general problems: example (c). Our theory can easily extend to problems with a potential function $q \in L^{\infty}(D)$. Garding's inequality holds for the bilinear form, and thus all theoretical results follow by the same argument. The normalized $L^{2}(D)$ - and $\widetilde{H}^{\alpha / 2}(D)$-norms of the spatial error are reported in Table 10 at $t=1$ for $\alpha=1.25,1.5$ and 1.75 . The results concur with the preceding convergence rates. 
TABLE 6. $L^{2}$ - and $\tilde{H}^{\alpha / 2}$-norms of the error for example (b1), nonsmooth initial data, with $\alpha=1.5$ for backward Euler method and $\tau=10^{-5}$.

\begin{tabular}{|c|c|cccccc|c|}
\hline$t$ & $h$ & $1 / 16$ & $1 / 32$ & $1 / 64$ & $1 / 128$ & $1 / 256$ & $1 / 512$ & ratio \\
\hline 0.1 & $L^{2}$ & $3.64 \mathrm{e}-3$ & $1.53 \mathrm{e}-3$ & $7.42 \mathrm{e}-4$ & $3.72 \mathrm{e}-5$ & $1.87 \mathrm{e}-4$ & $9.46 \mathrm{e}-5$ & $\approx 1.04(0.50)$ \\
\cline { 2 - 8 } & $H^{\alpha / 2}$ & $7.00 \mathrm{e}-2$ & $5.59 \mathrm{e}-2$ & $4.62 \mathrm{e}-2$ & $3.87 \mathrm{e}-2$ & $3.21 \mathrm{e}-2$ & $2.61 \mathrm{e}-2$ & $\approx 0.28(0.25)$ \\
\hline 0.01 & $L^{2}$ & $2.81 \mathrm{e}-2$ & $7.07 \mathrm{e}-2$ & $1.63 \mathrm{e}-3$ & $3.84 \mathrm{e}-4$ & $9.21 \mathrm{e}-5$ & $2.18 \mathrm{e}-5$ & $\approx 2.07(0.50)$ \\
\cline { 2 - 8 } & $H^{\alpha / 2}$ & $4.04 \mathrm{e}-1$ & $1.56 \mathrm{e}-1$ & $6.09 \mathrm{e}-2$ & $2.49 \mathrm{e}-2$ & $1.03 \mathrm{e}-2$ & $4.27 \mathrm{e}-3$ & $\approx 1.31(0.25)$ \\
\hline 0.005 & $L^{2}$ & $4.27 \mathrm{e}-2$ & $1.45 \mathrm{e}-2$ & $3.44 \mathrm{e}-3$ & $7.95 \mathrm{e}-4$ & $1.88 \mathrm{e}-4$ & $4.41 \mathrm{e}-4$ & $\approx 2.07(0.50)$ \\
\cline { 2 - 7 } & $H^{\alpha / 2}$ & $5.94 \mathrm{e}-1$ & $3.34 \mathrm{e}-1$ & $1.27 \mathrm{e}-1$ & $5.05 \mathrm{e}-2$ & $2.08 \mathrm{e}-2$ & $8.56 \mathrm{e}-3$ & $\approx 1.26(0.25)$ \\
\hline 0.001 & $L^{2}$ & $1.41 \mathrm{e}-1$ & $5.22 \mathrm{e}-2$ & $1.64 \mathrm{e}-2$ & $4.47 \mathrm{e}-3$ & $1.02 \mathrm{e}-3$ & $2.32 \mathrm{e}-3$ & $\approx 1.80(0.50)$ \\
\cline { 2 - 7 } & $H^{\alpha / 2}$ & $2.61 \mathrm{e} 0$ & $1.45 \mathrm{e} 0$ & $6.63 \mathrm{e}-1$ & $2.81 \mathrm{e}-1$ & $1.08 \mathrm{e}-1$ & $4.34 \mathrm{e}-2$ & $\approx 1.20(0.25)$ \\
\hline
\end{tabular}
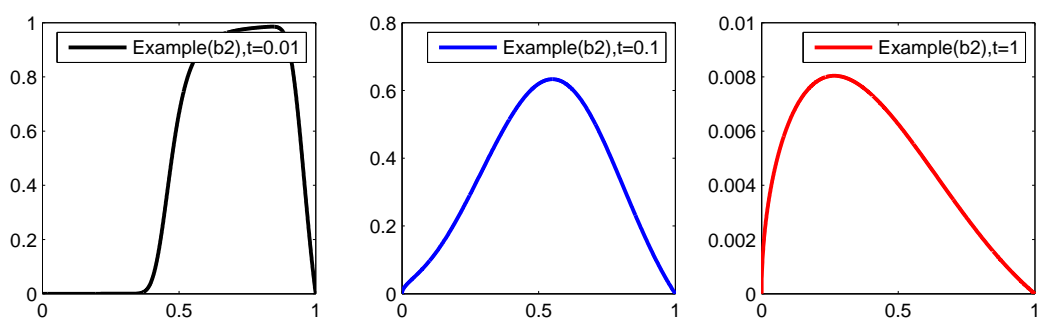

Figure 3. Solution profile of example (b1) with $\alpha=1.5$ at $0.01,0.1$ and 1.

TABLE 7. $L^{2}$ - and $\tilde{H}^{\alpha / 2}$-norms of the error for example (b2), nonsmooth initial data, for backward Euler method with $\tau=10^{-5}$.

\begin{tabular}{|c|c|cccccc|c|}
\hline$\alpha$ & $h$ & $1 / 16$ & $1 / 32$ & $1 / 64$ & $1 / 128$ & $1 / 256$ & $1 / 512$ & ratio \\
\hline \multirow{2}{*}{1.25} & $L^{2}$ & $6.31 \mathrm{e}-3$ & $3.55 \mathrm{e}-3$ & $2.07 \mathrm{e}-3$ & $1.23 \mathrm{e}-3$ & $7.38 \mathrm{e}-4$ & $4.53 \mathrm{e}-4$ & $\approx 0.76(0.25)$ \\
\cline { 2 - 7 } & $H^{\alpha / 2}$ & $6.03 \mathrm{e}-2$ & $5.37 \mathrm{e}-2$ & $4.86 \mathrm{e}-2$ & $4.42 \mathrm{e}-2$ & $4.02 \mathrm{e}-2$ & $3.67 \mathrm{e}-2$ & $\approx 0.14(0.13)$ \\
\hline \multirow{2}{*}{1.5} & $L^{2}$ & $4.11 \mathrm{e}-4$ & $1.91 \mathrm{e}-4$ & $9.24 \mathrm{e}-5$ & $4.55 \mathrm{e}-5$ & $2.26 \mathrm{e}-5$ & $1.12 \mathrm{e}-5$ & $\approx 1.03(0.50)$ \\
\cline { 2 - 7 } & $H^{\alpha / 2}$ & $7.88 \mathrm{e}-3$ & $6.48 \mathrm{e}-3$ & $5.39 \mathrm{e}-3$ & $4.48 \mathrm{e}-3$ & $3.70 \mathrm{e}-3$ & $3.01 \mathrm{e}-3$ & $\approx 0.27(0.25)$ \\
\hline \multirow{2}{*}{1.75} & $L^{2}$ & $2.75 \mathrm{e}-5$ & $1.21 \mathrm{e}-6$ & $5.09 \mathrm{e}-6$ & $2.11 \mathrm{e}-6$ & $8.48 \mathrm{e}-7$ & $3.20 \mathrm{e}-7$ & $\approx 1.28(0.75)$ \\
\cline { 2 - 6 } & $H^{\alpha / 2}$ & $4.50 \mathrm{e}-4$ & $3.33 \mathrm{e}-4$ & $2.46 \mathrm{e}-4$ & $1.86 \mathrm{e}-4$ & $1.39 \mathrm{e}-4$ & $1.01 \mathrm{e}-4$ & $\approx 0.42(0.38)$ \\
\hline
\end{tabular}

TABLE 8. $L^{2}$-norm of the error for example (b2), non-smooth initial data, with $h=2 \times 10^{-5}$ (BE - backward Euler, CN - Crank-Nicolson).

\begin{tabular}{|c|c|c|c|c|c|c|c|}
\hline & $\tau$ & $1 / 10$ & $1 / 20$ & $1 / 40$ & $1 / 80$ & $1 / 160$ & ratio \\
\hline \multirow[t]{3}{*}{$\mathrm{BE}$} & $\alpha=1.25$ & $3.57 \mathrm{e}-2$ & $1.71 \mathrm{e}-2$ & $8.09 \mathrm{e}-3$ & $3.80 \mathrm{e}-3$ & $1.71 \mathrm{e}-3$ & \multirow{3}{*}{$\begin{array}{l}\approx 1.09(1.00) \\
\approx 1.13(1.00) \\
\approx 1.20(1.00)\end{array}$} \\
\hline & $\alpha=1.5$ & $1.36 \mathrm{e}-2$ & $6.82 \mathrm{e}-3$ & $2.80 \mathrm{e}-3$ & $1.30 \mathrm{e}-3$ & $5.81 \mathrm{e}-4$ & \\
\hline & $\alpha=1.75$ & $4.55 \mathrm{e}-3$ & $1.78 \mathrm{e}-3$ & $7.57 \mathrm{e}-3$ & $3.35 \mathrm{e}-4$ & $1.48 \mathrm{e}-4$ & \\
\hline \multirow[t]{3}{*}{$\mathrm{CN}$} & $\alpha=1.25$ & $3.32 \mathrm{e}-3$ & $8.59 \mathrm{e}-4$ & $2.26 \mathrm{e}-4$ & $5.60 \mathrm{e}-5$ & $1.24 \mathrm{e}-5$ & \multirow{3}{*}{$\begin{array}{l}\approx 2.03(2.00) \\
\approx 1.99(2.00) \\
\approx 1.99(2.00)\end{array}$} \\
\hline & $\alpha=1.5$ & $9.36 \mathrm{e}-4$ & $2.59 \mathrm{e}-5$ & $6.95 \mathrm{e}-5$ & $1.74 \mathrm{e}-6$ & $3.80 \mathrm{e}-7$ & \\
\hline & $\alpha=1.75$ & $1.69 \mathrm{e}-4$ & $4.43 \mathrm{e}-5$ & $1.22 \mathrm{e}-5$ & $3.15 \mathrm{e}-6$ & $6.50 \mathrm{e}-7$ & \\
\hline
\end{tabular}

\section{CONCLUSION}

In this paper, we have studied a finite element method for an initial boundary value problem for the parabolic problem with a space fractional derivative of Riemann-Liouville type and order $\alpha \in(1,2)$ using the analytic semigroup theory. The existence and uniqueness of a weak solution in $L^{2}\left(0, T ; \widetilde{H}^{\alpha / 2}(D)\right)$ were established, and an improved regularity result was also shown. Error estimates in the $L^{2}(D)$ - and $\widetilde{H}^{\alpha / 2}(D)$-norm were established 
TABLE 9. $L^{2}$ - and $\tilde{H}^{\alpha / 2}$-norms of the error for example (b2), nonsmooth initial data, for backward Euler method with $\tau=10^{-5}$.

\begin{tabular}{|c|c|cccccc|c|}
\hline$t$ & $h$ & $1 / 16$ & $1 / 32$ & $1 / 64$ & $1 / 128$ & $1 / 256$ & $1 / 512$ & ratio \\
\hline 0.1 & $L^{2}$ & $1.73 \mathrm{e}-2$ & $8.56 \mathrm{e}-3$ & $4.27 \mathrm{e}-3$ & $2.14 \mathrm{e}-3$ & $1.08 \mathrm{e}-3$ & $5.43 \mathrm{e}-4$ & $\approx 1.00(0.50)$ \\
\cline { 2 - 8 } & $H^{\alpha / 2}$ & $3.83 \mathrm{e}-1$ & $3.20 \mathrm{e}-1$ & $2.67 \mathrm{e}-1$ & $2.23 \mathrm{e}-1$ & $1.84 \mathrm{e}-1$ & $1.50 \mathrm{e}-1$ & $\approx 0.26(0.25)$ \\
\hline 0.01 & $L^{2}$ & $3.35 \mathrm{e}-2$ & $1.39 \mathrm{e}-2$ & $6.45 \mathrm{e}-3$ & $3.17 \mathrm{e}-3$ & $1.58 \mathrm{e}-3$ & $7.97 \mathrm{e}-4$ & $\approx 1.07(0.50)$ \\
\cline { 2 - 8 } & $H^{\alpha / 2}$ & $6.41 \mathrm{e}-1$ & $4.89 \mathrm{e}-1$ & $3.97 \mathrm{e}-1$ & $3.28 \mathrm{e}-1$ & $2.71 \mathrm{e}-1$ & $2.20 \mathrm{e}-1$ & $\approx 0.30(0.25)$ \\
\hline 0.005 & $L^{2}$ & $4.23 \mathrm{e}-2$ & $1.83 \mathrm{e}-2$ & $7.65 \mathrm{e}-3$ & $3.61 \mathrm{e}-3$ & $1.79 \mathrm{e}-3$ & $8.96 \mathrm{e}-4$ & $\approx 1.11(0.50)$ \\
\cline { 2 - 7 } & $H^{\alpha / 2}$ & $7.52 \mathrm{e}-1$ & $5.89 \mathrm{e}-1$ & $4.55 \mathrm{e}-1$ & $3.71 \mathrm{e}-1$ & $3.04 \mathrm{e}-1$ & $2.47 \mathrm{e}-1$ & $\approx 0.29(0.25)$ \\
\hline 0.001 & $L^{2}$ & $1.07 \mathrm{e}-1$ & $4.12 \mathrm{e}-2$ & $1.54 \mathrm{e}-2$ & $5.89 \mathrm{e}-3$ & $2.49 \mathrm{e}-3$ & $1.19 \mathrm{e}-3$ & $\approx 1.30(0.50)$ \\
\cline { 2 - 7 } & $H^{\alpha / 2}$ & $1.98 \mathrm{e} 0$ & $1.19 \mathrm{e} 0$ & $7.51 \mathrm{e}-1$ & $5.19 \mathrm{e}-1$ & $4.08 \mathrm{e}-1$ & $3.28 \mathrm{e}-1$ & $\approx 0.52(0.25)$ \\
\hline
\end{tabular}

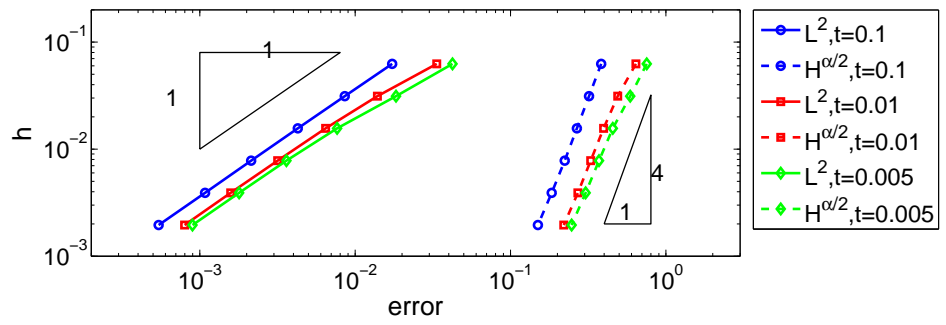

Figure 4. Numerical results for example (b2) with $\alpha=1.5$ at $t=$ $0.1,0.01$ and 0.005 .

TABLE 10. $L^{2}$-norm of the error for the general differential equation non-smooth initial data, example (c), with $\tau=2 \times 10^{-5}$ (BE - backward Euler, CN - Crank-Nicolson).

\begin{tabular}{|c|c|cccccc|c|}
\hline$\alpha$ & $h$ & $1 / 16$ & $1 / 32$ & $1 / 64$ & $1 / 128$ & $1 / 256$ & $1 / 512$ & ratio \\
\hline \multirow{2}{*}{1.25} & $L^{2}$ & $4.80 \mathrm{e}-3$ & $2.71 \mathrm{e}-3$ & $1.58 \mathrm{e}-3$ & $9.40 \mathrm{e}-4$ & $5.66 \mathrm{e}-4$ & $3.48 \mathrm{e}-4$ & $\approx 0.76(0.25)$ \\
\cline { 2 - 7 } & $H^{\alpha / 2}$ & $4.62 \mathrm{e}-2$ & $4.12 \mathrm{e}-2$ & $3.73 \mathrm{e}-2$ & $3.39 \mathrm{e}-2$ & $3.09 \mathrm{e}-2$ & $2.82 \mathrm{e}-2$ & $\approx 0.14(0.13)$ \\
\hline \multirow{2}{*}{1.5} & $L^{2}$ & $2.75 \mathrm{e}-4$ & $1.31 \mathrm{e}-4$ & $6.50 \mathrm{e}-5$ & $3.24 \mathrm{e}-5$ & $1.63 \mathrm{e}-5$ & $8.20 \mathrm{e}-5$ & $\approx 1.00(0.50)$ \\
\cline { 2 - 7 } & $H^{\alpha / 2}$ & $5.90 \mathrm{e}-3$ & $6.86 \mathrm{e}-3$ & $4.05 \mathrm{e}-3$ & $3.37 \mathrm{e}-3$ & $2.79 \mathrm{e}-3$ & $2.26 \mathrm{e}-3$ & $\approx 0.27(0.25)$ \\
\hline \multirow{2}{*}{1.75} & $L^{2}$ & $7.88 \mathrm{e}-6$ & $3.19 \mathrm{e}-6$ & $1.33 \mathrm{e}-6$ & $5.58 \mathrm{e}-7$ & $2.34 \mathrm{e}-7$ & $9.60 \mathrm{e}-8$ & $\approx 1.27(0.75)$ \\
\cline { 2 - 6 } & $H^{\alpha / 2}$ & $3.24 \mathrm{e}-4$ & $2.42 \mathrm{e}-4$ & $1.80 \mathrm{e}-4$ & $1.36 \mathrm{e}-4$ & $1.02 \mathrm{e}-4$ & $7.43 \mathrm{e}-5$ & $\approx 0.42(0.38)$ \\
\hline
\end{tabular}

for a space semidiscrete scheme with a piecewise linear finite element method, and $L^{2}(D)$ norm estimates for fully discrete schemes based on the backward Euler method and the Crank-Nicolson method, for both smooth and nonsmooth initial data.

The numerical experiments fully confirmed the convergence of the numerical schemes, but the $L^{2}(D)$-norm error estimates are suboptimal: the empirical convergence rates are one-half order higher than the theoretical ones. This suboptimality is attributed to the inefficiency of Nitsche's trick, as a consequence of the low regularity of the adjoint solution. Numerically, we observe that the $\widetilde{H}^{\alpha / 2}(D)$-norm convergence rates agree well with the theoretical ones. The optimal convergence rates in the $L^{2}(D)$-norm and the $\widetilde{H}^{\alpha / 2}(D)$ norm estimate for the fully discrete schemes still await theoretical justifications.

\section{ACKNOWLEDGEMENTS}

The research of B. Jin has been supported by NSF Grant DMS-1319052, R. Lazarov was supported in parts by NSF Grant DMS-1016525 and J. Pasciak has been supported by NSF Grant DMS-1216551. The work of all authors was also supported by Award No. KUS-C1-016-04, made by King Abdullah University of Science and Technology (KAUST). 


\section{Appendix A. Proof of Theorem 3.1}

Proof. We divide the proof into four steps.

Step (i) (energy estimates for $u_{m}$ ). Upon taking $u_{m}$ as the test function, the identity $2\left(u_{m}^{\prime}, u_{m}\right)=\frac{d}{d t}\left\|u_{m}\right\|_{L^{2}(D)}^{2}$ for a.e. $0 \leq t \leq T$, and the coercivity of $A(\cdot, \cdot)$, we deduce

$$
\frac{d}{d t}\left\|u_{m}(t)\right\|_{L^{2}(D)}^{2}+c_{0}\left\|u_{m}(t)\right\|_{\widetilde{H}^{\alpha / 2}(D)}^{2} \leq 2\|f(t)\|_{H^{-\alpha / 2}(D)}\left\|u_{m}(t)\right\|_{\widetilde{H}^{\alpha / 2}(D)} .
$$

Young's inequality and integration in $t$ over $(0, t)$ gives

$$
\max _{0 \leq t \leq T}\left\|u_{m}(t)\right\|_{L^{2}(D)}^{2} \leq\|v\|_{L^{2}(D)}^{2}+C\|f\|_{L^{2}\left(0, T ; H^{-\alpha / 2}(D)\right)}^{2} .
$$

Next we integrate A.1 from 0 to $T$, and repeat the argument to get

$$
\left\|u_{m}\right\|_{L^{2}\left(0, T ; \widetilde{H}^{\alpha / 2}(D)\right)}^{2} \leq\|v\|_{L^{2}(D)}^{2}+C\|f\|_{L^{2}\left(0, T ; H^{-\alpha / 2}(D)\right)}^{2} .
$$

Finally we bound $\left\|u_{m}^{\prime}\right\|_{L^{2}\left(0, T ; H^{-\alpha / 2}(D)\right)}$. For any $\varphi \in \widetilde{H}^{\alpha / 2}(D)$ such that $\|\varphi\|_{\widetilde{H}^{\alpha / 2}(D)} \leq$ 1, we decompose it into $\varphi=P \varphi+(I-P) \varphi$ with $P \varphi \in \operatorname{span}\left\{\omega_{k}\right\}_{k=1}^{m}$ and $I-P \in$ $\operatorname{span}\left\{\omega_{k}\right\}_{k>m}$. By the stability of the projection $P,\|P \varphi\|_{\widetilde{H}^{\alpha / 2}(D)} \leq C\|\varphi\|_{\widetilde{H}^{\alpha / 2}(D)} \leq C$, it follows from $\left(u_{m}^{\prime}, P \varphi\right)+A\left(u_{m}, P \varphi\right)=(f, P \varphi)$ and $\left(u_{m}^{\prime}, P \varphi\right)=\left(u_{m}^{\prime}, \varphi\right)$ that

$$
\left|\left\langle u_{m}^{\prime}(t), \varphi\right\rangle\right|=\left|\left\langle u_{m}^{\prime}(t), P \varphi\right\rangle\right| \leq C\left(\|f(t)\|_{H^{-\alpha / 2}(D)}+\left\|u_{m}(t)\right\|_{\widetilde{H}^{\alpha / 2}(D)}\right) .
$$

Consequently, by the duality argument and (A.2) we arrive at

$$
\left\|u_{m}^{\prime}\right\|_{L^{2}\left(0, T ; H^{-\alpha / 2}(D)\right)}^{2} \leq C\left(\|f\|_{L^{2}\left(0, T ; H^{-\alpha / 2}(D)\right)}^{2}+\|v\|_{L^{2}(D)}^{2}\right) .
$$

Step (ii) (convergent subsequence). By A.2 and A.3 there exists a subsequence, also denoted by $\left\{u_{m}\right\}$, and $u \in L^{2}\left(0, T ; \widetilde{H}^{\alpha / 2}(D)\right)$ and $\tilde{u} \in L^{2}\left(0, T ; H^{-\alpha / 2}(D)\right)$, such that

$$
\begin{aligned}
& u_{m} \rightarrow u \quad \text { weakly in } L^{2}\left(0, T ; \widetilde{H}^{\alpha / 2}(D)\right), \\
& u_{m}^{\prime} \rightarrow \tilde{u} \quad \text { weakly in } L^{2}\left(0, T ; H^{-\alpha / 2}(D)\right) .
\end{aligned}
$$

By choosing $\phi \in C_{0}^{\infty}[0, T]$ and $\psi \in \widetilde{H}^{\alpha / 2}(D)$, we deduce

$$
\int_{0}^{T}\left\langle u_{m}^{\prime}, \phi \psi\right\rangle d t=-\int_{0}^{T}\left\langle u_{m}, \phi^{\prime} \psi\right\rangle d t
$$

By taking $m \rightarrow \infty$ we obtain

$$
\int_{0}^{T}\langle\tilde{u}, \phi \psi\rangle d t=-\int_{0}^{T}\left\langle u, \phi^{\prime} \psi\right\rangle d t=\int_{0}^{T}\left\langle u^{\prime}, \phi \psi\right\rangle d t .
$$

Thus $\tilde{u}=u^{\prime}$ by the density of $\{\phi(t) \psi(x)\}$ in $L^{2}\left(0, T ; \widetilde{H}^{\alpha / 2}(D)\right)$.

Step (iii) (weak form). Now for a fixed integer $N$, we choose a test function $\psi \in V_{N}=$ $\operatorname{span}\left\{\omega_{k}\right\}_{k=1}^{N}$, and $\phi \in C^{\infty}[0, T]$. Then for $m \geq N$, there holds

$$
\int_{0}^{T}\left\langle u_{m}^{\prime}, \psi \phi\right\rangle+A\left(u_{m}, \psi\right) \phi d t=\int_{0}^{T}\langle f, \psi \phi\rangle d t .
$$

Then letting $m \rightarrow \infty$, A.4 and the density of $\{\phi(t) \psi(x)\}$ in $L^{2}\left(0, T ; \widetilde{H}^{\alpha / 2}(D)\right)$ gives

$$
\int_{0}^{T}\left\langle u^{\prime}, \varphi\right\rangle+A(u, \varphi) d t=\int_{0}^{T}\langle f, \varphi\rangle d t, \quad \forall \varphi \in L^{2}\left(0, T ; \widetilde{H}^{\alpha / 2}(D)\right) .
$$

Consequently, we arrive at

$$
\left\langle u^{\prime}, \varphi\right\rangle+A(u, \varphi)=\langle f, \varphi\rangle, \quad \forall \varphi \in \widetilde{H}^{\alpha / 2}(D) \quad \text { a.e. } 0 \leq t \leq T .
$$

(iv) (initial condition). The argument presented in [4, Theorem 3, pp. 287] yields $u \in C\left([0, T] ; L^{2}(D)\right)$. By taking $\phi \in C^{\infty}[0, T]$ with $\varphi(T)=0$ and $\psi \in \operatorname{span}\left\{\omega_{k}\right\}_{k=1}^{N}$, integrating A.5 and a.6 by parts with respect to $t$, and a standard density argument, 
we arrive at the initial condition $u(0)=v$. The uniqueness follows directly from the energy estimates.

\section{REFERENCES}

[1] D. A. Benson, S. W. Wheatcraft, and M. M. Meerschaert. The fractional-order governing equation of Lévy motion. Water Resour. Res., 36(6):1413-1424, 2000.

[2] V. J. Ervin and J. P. Roop. Variational formulation for the stationary fractional advection dispersion equation. Numer. Methods Partial Diff. Eq., 22(3):558-576, 2006.

[3] V. J. Ervin and J. P. Roop. Variational solution of fractional advection dispersion equations on bounded domains in $\mathbb{R}^{d}$. Numer. Methods Partial Diff. Eq., 23(2):256-281, 2007.

[4] L. C. Evans. Partial Differential Equations. AMS, Providence, RI, 2010.

[5] H. Fujita and A. Mizutani. On the finite element method for parabolic equations. I. Approximation of holomorphic semi-groups. J. Math. Soc. Japan, 28(4):749-771, 1976.

[6] H. Fujita and T. Suzuki. Evolution problems. In Handbook of Numerical Analysis, Vol. II, Handbook Numer. Anal., II, pages 789-928. North-Holland, Amsterdam, 1991.

[7] A. Hansbo. Nonsmooth data error estimates for damped single step methods for parabolic equations in Banach space. Calcolo, 36(2):75-101, 1999.

[8] M. Hasse. The Functional Calculus for Sectorial Operators. Birkhauser-Verlag, 2006

[9] Y. Hatano and N. Hatano. Dispersive transport of ions in column experiments: An explanation of long-tailed profiles. Water Res. Research, 34(5):1027-1033, 1998.

[10] K. Ito and F. Kappel. Evolutions Equations and Approximations, volume 16 of Anvances in Mathematics for Applied Sciences. World Scientific, 2002.

[11] B. Jin, R. Lazarov, and J. Pasciak. Variational formulation of problems involving fractional order differential operators. preprint, 2013.

[12] A. Kilbas, H. Srivastava, and J. Trujillo. Theory and Applications of Fractional Differential Equations. Elsevier, Amsterdam, 2006.

[13] M. Luskin and R. Rannacher. On the smoothing property of the Crank-Nicolson scheme. Applicable Anal., 14(2):117-135, 1974

[14] R. Metzler and J. Klafter. The random walk's guide to anomalous diffusion: a fractional dynamics approach. Phys. Rep., 339(1):1-77, 2000.

[15] A. Pazy. Semigroup of Linear Operators and Applications to Partial Differential Equations. Springer-Verlag, 1983.

[16] C. Tadjeran and M. M. Meerschaert. A second-order accurate numerical method for the twodimensional fractional diffusion equation. J. Comput. Phys., 220(2):813-823, 2007.

[17] C. Tadjeran, M. M. Meerschaert, and H.-P. Scheffler. A second-order accurate numerical approximation for the fractional diffusion equation. J. Comput. Phys., 213(1):205-213, 2006.

[18] V. Thomée. Galerkin Finite Element Methods for Parabolic Problems. Springer-Verlag, Berlin, 2006.

[19] M. Zlámal. Finite element methods for parabolic equations. Math. Comp., 28(126):393-404, 1974.

Department of Mathematics, University of California, Riverside, University Ave. 900, Riverside, CA 92521 (bangti. Jin@gmail.com)

Department of Mathematics, Texas A\&M University, College Station, TX 77843-3368 (lazarov, PASCIAK, ZZHOU@MATH.TAMU.EDU) 